Original Full Length Article

\title{
FGF23-regulated production of Fetuin-A (AHSG) in osteocytes
}

\author{
D. Mattinzoli a , M.P. Rastaldi a , M. Ikehata ${ }^{a}$, S. Armelloni ${ }^{\text {a }}$, C. Pignatari ${ }^{\text {a }}$, L.A. Giardino ${ }^{\text {a }}$, M. Li ${ }^{\text {a }}$, C.M. Alfieri ${ }^{\text {a }}$, \\ A. Regalia ${ }^{a}$, D. Riccardi ${ }^{\text {b }}$, P. Messa ${ }^{\mathrm{a}, *}$ \\ ${ }^{a}$ Renal Research Laboratory, Fondazione IRCCS Ca' Granda Ospedale Maggiore Policlinico E' Fondazione D'Amico per la Ricerca sulle Malattie Renali, via Pace 9, 20122 Milano, Italy \\ ${ }^{\mathrm{b}}$ Division of Pathophysiology and Repair, School of Biosciences, Cardiff University, Cardiff, UK
}

\section{A R T I C L E I N F O}

Article history:

Received 9 July 2015

Revised 24 September 2015

Accepted 13 October 2015

Available online 23 October 2015

\section{Keyword:}

Osteocyte

AHSC

FGF23

FGF receptor

Chronic kidney disease

Vascular calcification

\begin{abstract}
A B S T R A C T
Introduction: AHSG, a serum glycoprotein with recognized anti-calcification activity, has also been suggested to modulate both bone formation and resorption. Though the bulk of AHSG is mostly synthesized in the liver, it has been claimed that also bone cells might produce it. However, the extent of the bone AHSG production and the potential controlling factors remain to be definitively proven.

A relevant number of studies support the notion that FGF23, a bone-derived hormone, not only regulates the most important mineral metabolism (MM) related factors (phosphate, parathyroid hormone, vitamin $\mathrm{D}$, etc.), but might be also involved in cardiovascular (CV) outcome, both in chronic kidney disease (CKD) patients and in the general population. Furthermore, in addition to some direct autocrine and paracrine effects in bone, FGF23 has been suggested to interact with AHSG.

In this study we investigated if AHSG is really produced by bone cells, and if its bone production is related and/or controlled by FGF23, using cultured bone cells, according to a new method recently published by our group. Results: Our data show that AHSG is consistently produced in osteocytes and to a far lesser extent in osteoblasts. Both FGF23 addition to the culture medium and its over-expression in osteocytes were associated with a consistent increase of both AHSG mRNA and protein, while FGF23 silencing was followed by opposite effects. Though most of these results were largely affected by the blockage of FGF23 receptors, the role of these receptors in the different experimental sets is still not completely clarified. In addition, we found that FGF23 and AHSG proteins co-localized both in cytoplasm and nucleus, which suggests a possible reciprocal interactivity.

Conclusions: Our data not only confirm that AHSG is produced in bone, mainly in osteocytes, but show for the first time that its production is modulated by FGF23. Since both proteins play important roles in the bone and cardiovascular pathology, these results add new pieces to the puzzling relationship between bone and vascular pathology, in particular in CKD patients, prompting future investigations in this field.
\end{abstract}

(c) 2015 Elsevier Inc. All rights reserved.

\section{Introduction}

AHSG (alpha2-HS-glycoprotein), a reverse acute phase serum glycoprotein (MW $52 \mathrm{kDa}$ ), belonging to the family of the type 3 cystatin proteins, was first described by Pedersen more than 7 decades ago [1]. It has long been known that AHSG, thanks to its exceedingly high content in

Abbreviations: AHSG, Fetuin A; MM, mineral metabolism; CKD, chronic kidney disease; Os, osteocytes; Ob, osteoblasts; Oc, osteoclasts; CV, cardiovascular; TF, transcription factor; WB, western blot; FGFR, FGF receptor; IS, immunostaining; IHC, immunochemistry.

* Corresponding author.

E-mail addresses: deborah.mattinzoli@gmail.com (D. Mattinzoli), mariapia.rastaldi@policlinico.mi.it (M.P. Rastaldi), aquamarine0321@libero.it (M. Ikehata), armellonis@libero.it (S. Armelloni), chiarapignatari@gmail.com (C. Pignatari), giardinolaura@gmail.com (L.A. Giardino), li_min@libero.it (M. Li), carlo.alfieri1@gmail.com (C.M. Alfieri), anna.regalia1@gmail.com (A. Regalia), riccardi@cardiff.ac.uk (D. Riccardi), pmessa@policlinico.mi.it (P. Messa). acidic amino-acids, is characterized by particularly high mineral affinity and, in addition to its circulating pool, is consistently represented in the mineralized bone matrix [2,3] and in ectopic calcifications [4], where it mainly acts as an anti-calcification factor [5,6]. Furthermore, it is known that AHSG, which presents homology 1 domain with TGF- $\beta$-receptor II, has been demonstrated in experimental studies to modulate osteogenesis TGF- $\beta$-mediated [7].

Overall, much experimental evidence has demonstrated that AHSG, which constitutes $25 \%$ of non-collagenous proteins in bone, could efficiently inhibit mineralization and control both bone formation and resorption [8-9]. Since the bulk of circulating AHSG is mostly synthesized in the liver, it was initially assumed that the protein in the bone was mainly, if not exclusively, derived from the circulating pool [10, 11 ], though a subsequent study suggested that osteoblasts (Ob) express AHSG mRNA and protein and that at least part of AHSG present in bone is derived from bone cells [12,13]. However, the real extent and the potential controlling factors on the possible AHSG production by bone cells are still not fully defined. 
Recently, a significant number of studies have shown that FGF23, a $32 \mathrm{kDa}$ protein synthesized by bone cells (osteoblasts/osteocytes), is a bone-derived hormone mainly devoted to regulate the most important mineral metabolism (MM) related factors (phosphate, parathyroid hormone, vitamin D, etc.) $[14,15,16]$. The main recognized stimuli for FGF23 production are high phosphorus intake/levels and/or vitamin D activity [17]. Its main effects are mediated by high-affinity binding to the FGF-receptor/ $\alpha$-Klotho complex and translate into inhibition of phosphate renal tubular transport, calcitriol levels (by both inhibition of synthesis and increase of VitD catabolism) and reduction in PTH secretion $[17,18,19]$.

Furthermore, both experimental and clinical studies suggest that, in addition to the well known MM related effects, high FGF23 levels could be related to poor cardiovascular (CV) outcomes, both in chronic kidney disease (CKD) patients and in the general population [20]. Moreover, FGF23 can also have direct autocrine and paracrine effects in bone which could be in some way linked to AHSG production itself. Furthermore some preliminary evidence suggests that FGF23 and AHSG might interact also at the vascular level, with a potential fall-out on the vascular calcification process, particularly in CKD [13,21].

Finally, osteocytes (Os) have been increasingly recognized as the central cells in the production of the main bone derived factors acting either as hormonal substance and/or autocrine factors regulating bone metabolism itself $[22,23]$. We recently described a new efficient method for the in vitro production of well-functioning and characterized Os, which clearly produce both sclerostin and FGF23 [24,25,26].

On the basis of these considerations, we used the Os in vitro system already described in our previous study to investigate: if AHSG is really produced by Os, and if its secretion, if any, could be in some way related to and/or controlled by FGF23.

\section{Results}

\subsection{FGF23 expression in mice bone tissue and in cultured $\mathrm{Ob}$ and $\mathrm{Os}$}

Immunochemistry (IHC) performed on tibial bone of Balb/c mice shows that FGF23 was highly expressed in Os (Fig. 1A). On the other hand, an only marginal immunostaining was evident in the mature osteoblasts lining the bone marrow space where highly positive bone cells are evident (Fig. 1B). The lower magnification figure gives information about the analyzed tissue area (Fig. 1C).

In ATRA-cultured cells, FGF23 expression progressively increased during Os maturation as assessed by both IS and WB (Fig. 1D-G). Quantitative Real Time RT-PCR (qRT PCR) also confirmed a significant increase of FGF23 mRNA expression in Os (Fig. 1H). On the contrary, the levels of FGF23 protein (data not shown) and mRNA (Fig. 1I) progressively decreased in $\mathrm{Ob}$ and were consistently lower than in Os at the time 12 (Fig. 1L).

\subsection{AHSG expression in mice bone tissue and in cultured $\mathrm{Ob}$ and $\mathrm{Os}$}

Os of mice tibial bone highly expressed AHSG protein as assessed by IHC in Os (Fig. 2A) and marginally in Ob (Fig. 2B). The lower magnification gives an idea of the tissue areas analyzed (Fig. 2C).

AHSG expression increased in a time-dependent way in cultured Os as shown by IS (Fig. 2D, E, F), WB (Fig. 2G) and qRT PCR (Fig. 2H). On the other hand, AHSG protein (data not shown) and mRNA expression progressively decreased in Ob (Fig. 2I). Overall, AHSG expression was by far lower in Ob than Os and almost undetectable in the former cells after 12 days in culture (Fig. 2L).
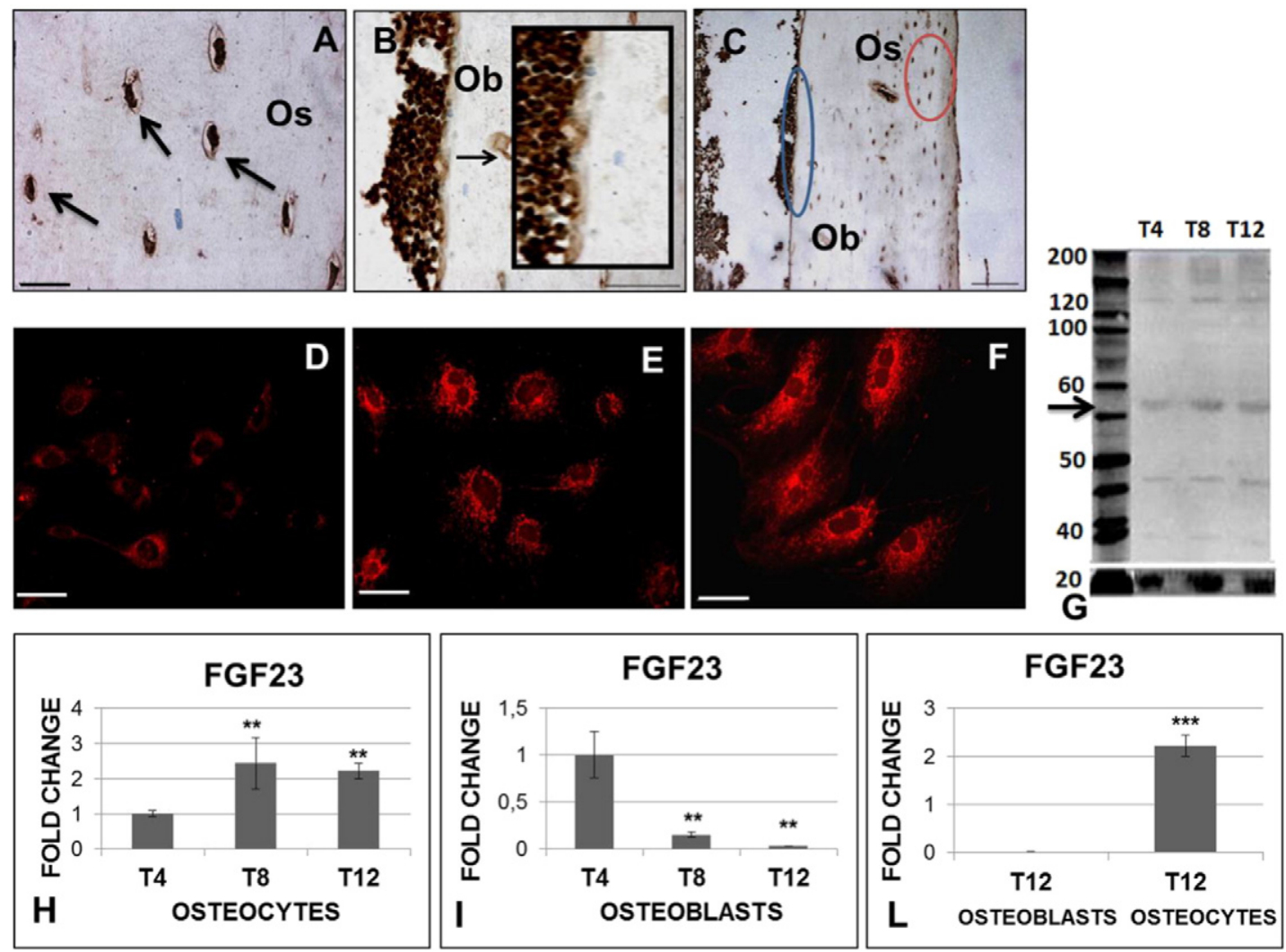

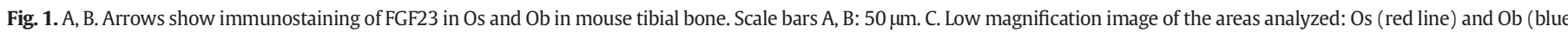

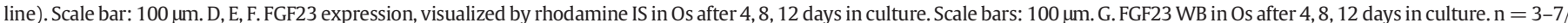

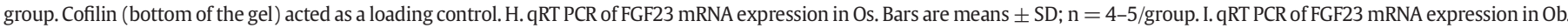

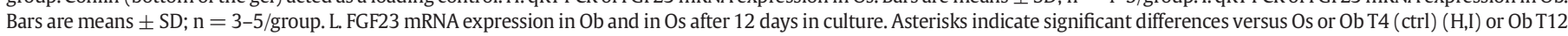
$(\mathrm{L}):^{* *}=\mathrm{p}<0.01,{ }^{* * *}=\mathrm{p}<0.001$ by ANOVA. 

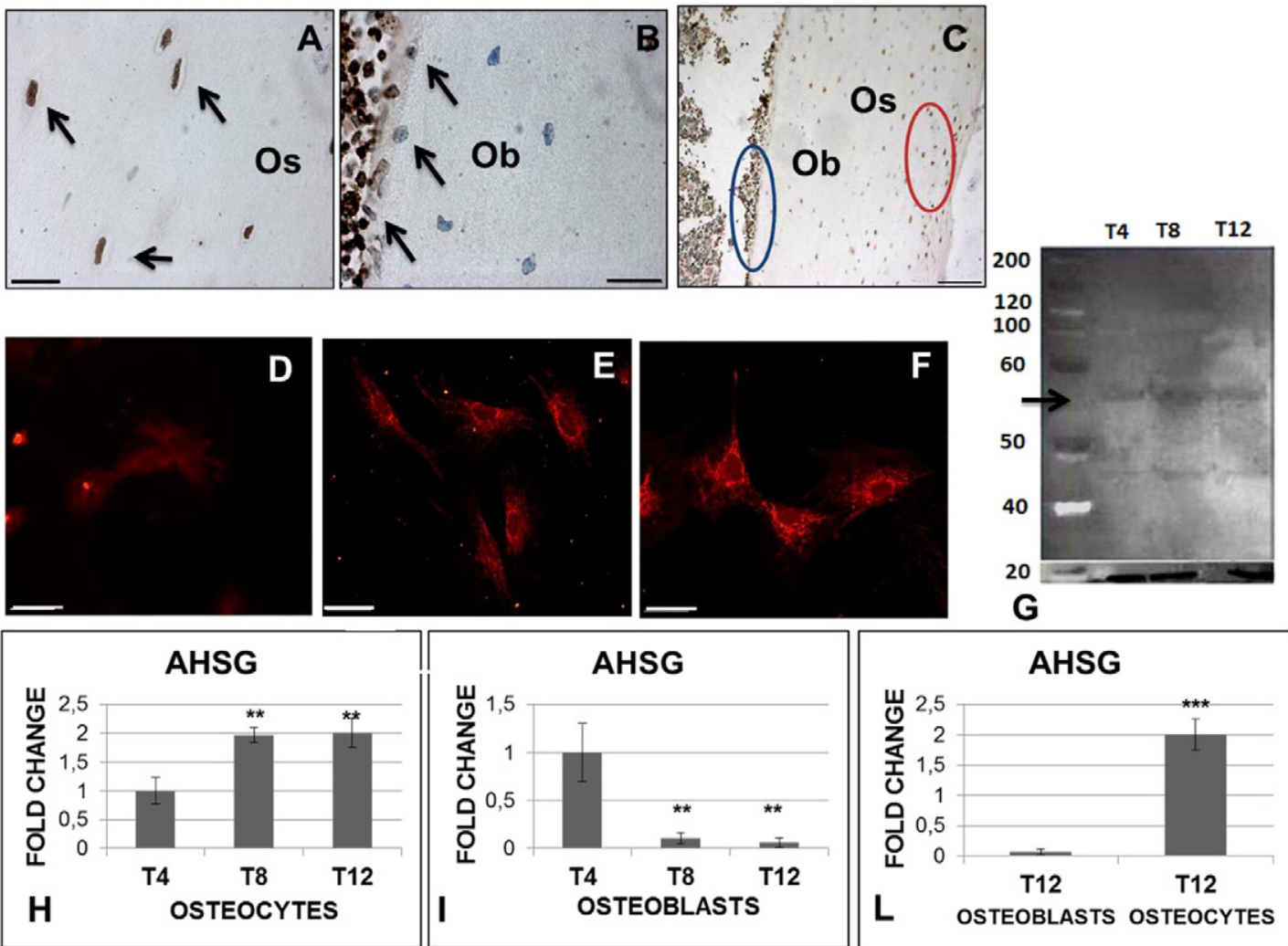

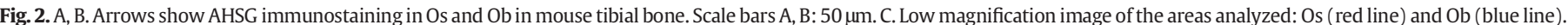

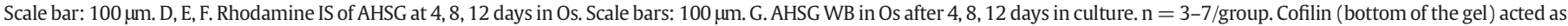

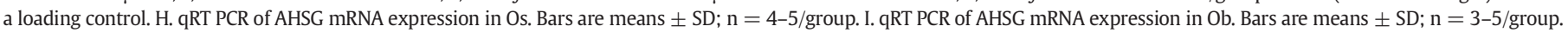
L. AHSG mRNA expression at 12 days in Ob and in Os. Asterisks indicate significant differences versus Os or Ob T4 (ctrl) $(\mathrm{H}, \mathrm{I})$ or $\mathrm{Ob} \mathrm{T} 12(\mathrm{~L})$ : ${ }^{* *}=\mathrm{p}<0.01$, ${ }^{* * *}=\mathrm{p}<0.001 \mathrm{by} \mathrm{ANOVA}$.

\subsection{Release of AHSG and FGF23 protein in the medium of cultured $O b$ and} Os

FGF23 released in the cell-culture medium progressively increased over time in Os whereas in Ob the observed release was lower and stable (Fig. 3A). Quantitative analysis of AHSG by ELISA showed a far greater secretion of the protein by Os as compared to the minimal amount produced by Ob (Fig. 3B).

\subsection{Interactions between AHSG and FGF23}

In order to investigate the possibility that FGF23 and AHSG might interact with one other, we focussed our studies on Os cells since these cells maximally express these proteins from T4 onward, where T4 represents the beginning of the differential expression of the two proteins of interested.

\subsubsection{Reciprocal effects of the addition of FGF23 or AHSG}

mRNA expression of both FGF23 and AHSG significantly increased $24 \mathrm{~h}$ after the addition of recombinant FGF23. On the contrary, AHSG addition did not affect the expression of either of the two (Fig. 4A, B).

\subsubsection{Effects of recombinant FGF23 addition on AHSG expression at differ- ent culture times}

In order to explore at which time in culture the FGF23 stimulatory action on AHSG was maximal, we evaluated the changes of AHSG mRNA expression after FGF23 addition for $24 \mathrm{~h}$ in cells cultured for 4 , 8,12 days. AHSG mRNA expression was confirmed to increase with the advancing of time in culture (Fig. 5A). However, FGF23 addition seemed to significantly affect AHSG mRNA expression only at day 4 .
These data were also confirmed by both the protein expression (Fig. 5B, C) and qualitative WB (Fig. 5D). Moreover, a very evident more elongated shape of the Os treated with FGF23 was observed (Fig. 5E, F). In contrast, no difference in cell morphology was observed in the cells treated with AHSG (data not shown).

\subsubsection{Effect of FGF23 gene overexpression on AHSG expression.}

To assess if not only the addition but also FGF23 overexpression could affect the AHSG production, we transfected T4 Os with mouse cDNA FGF23. Transfection induced a more than tenfold increase in FGF23 mRNA (Fig. 6A) which was associated with a more than doubling of AHSG mRNA levels after $48 \mathrm{~h}$ (Fig. 6B). Immunofluorescence (Fig. 6C, D) and WB (Fig. 6E) confirmed an increase also of the AHSG protein levels. Consistent with findings reported above, found a similar, but more evident elongated shape in the Os overexpressing FGF23 (Fig. 6F, G).

\subsubsection{Effects of FGF23 gene silencing on AHSG expression}

To test the hypothesis that FGF23 reduction might also affect AHSG levels, we transfected T4 Os with FGF23 specific siRNA.

qRT PCR confirmed an almost complete FGF23 mRNA silencing at $48 \mathrm{~h}$ (Fig. 7A), and a relatively marked reduction of both AHSG mRNA (Fig. 7B) and protein (Fig. 7C, D) in Os. Qualitative WB analyses at $48 \mathrm{~h}$ confirmed AHSG protein abolition in silenced Os (Fig. 7E).

\subsection{FGF receptor (FGFR) expression and effects of their inhibitor on FGF23}

\subsubsection{FGFR expression in cultured Os}

To investigate if the stimulatory effects of either FGF23 addition or overexpression on AHSG production were mediated by FGFR, we explored first whether our cultured cells expressed the known FGFRs. 

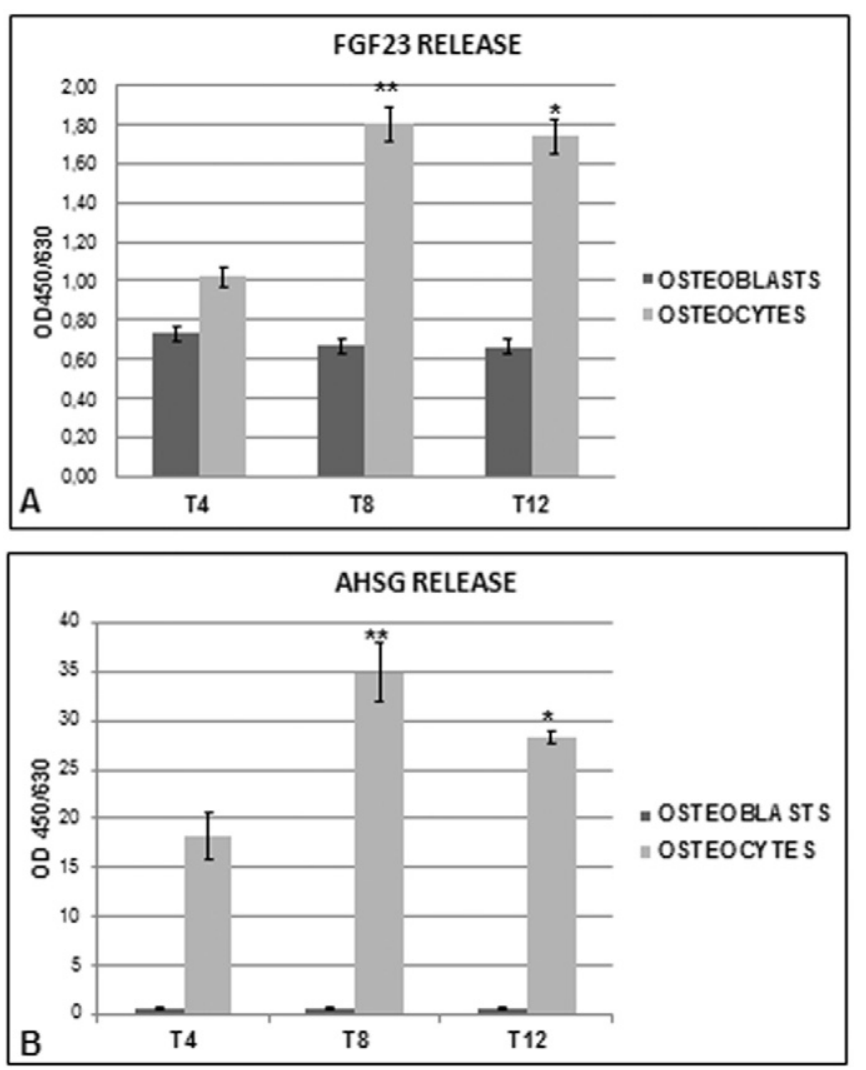

Fig. 3. A. Cultured medium harvested after $\mathrm{Ob}$ and Os were cultured for 4, 8, 12 days for measurements of FGF23 protein content, assessed by ELISA. Bars are means \pm SD; $\mathrm{n}=$ 3/group. B. Cultured medium harvested after Ob and Os were cultured for 4, 8, 12 days for measurements of AHSG protein content, assessed by ELISA. Bars are means $\pm \mathrm{SD}$; $\mathrm{n}=3$ /group. Data were normalized by Janus Green Nuclear Marker. Asterisks indicate significant differences versus Os T4 (ctrl): ${ }^{*}=\mathrm{p}<0.05,{ }^{* *}=\mathrm{p}<0.01$ by ANOVA.

Indeed, FGFR1, FGFR2, FGFR3 and FGFR4, evaluated by semi quantitative RT-PCR (Fig. 8A) were all clearly present in cultured Os, though the FGFR1 appeared more brightly expressed than the others.

\subsubsection{Influence of FGFR inhibition on FGF23 expression}

Then, we explored whether blocking FGFR by FIIN1 (a global and irreversible FGFRs blocker) affects the FGF23 auto-stimulatory effects secondary to the addition of FGF23 to the culture medium. Addition of FIIN1 alone to Os did not induce any change of FGF23 mRNA and protein expression levels (Fig. 8B, C). Furthermore, the simultaneous addition of FIIN1 and recombinant FGF23 completely abolished the autostimulatory effects of the external FGF23 addition (Fig. 8B, C).

\subsubsection{Influence of FGFRs inhibition on AHSG expression}

Next we explored the effects of FGFRs blockade on the AHSG stimulating effects by FGF23 addition. As in the previous experiments, FGF23 addition confirmed its stimulating effects on AHSG mRNA expression (Fig. 9A). Unexpectedly, FGFRs block alone resulted in a stimulating effect on AHSG mRNA (Fig. 9A), confirmed by a parallel increase of protein expression, detected by qualitative WB (Fig. 9B). When recombinant FGF23 was added in the presence of FGFRs block to the cultured Os, a further significant increase in mRNA and, to a lesser extent of the protein production were observed (Fig. 9B).

\subsection{Effects of FGF23 overexpression with the contemporary FGFRs block, on AHSG production}

We then tested the effects of FGF23 overexpression in the presence of FIIN1. The FGF23 overexpression reconfirmed a stimulatory effect
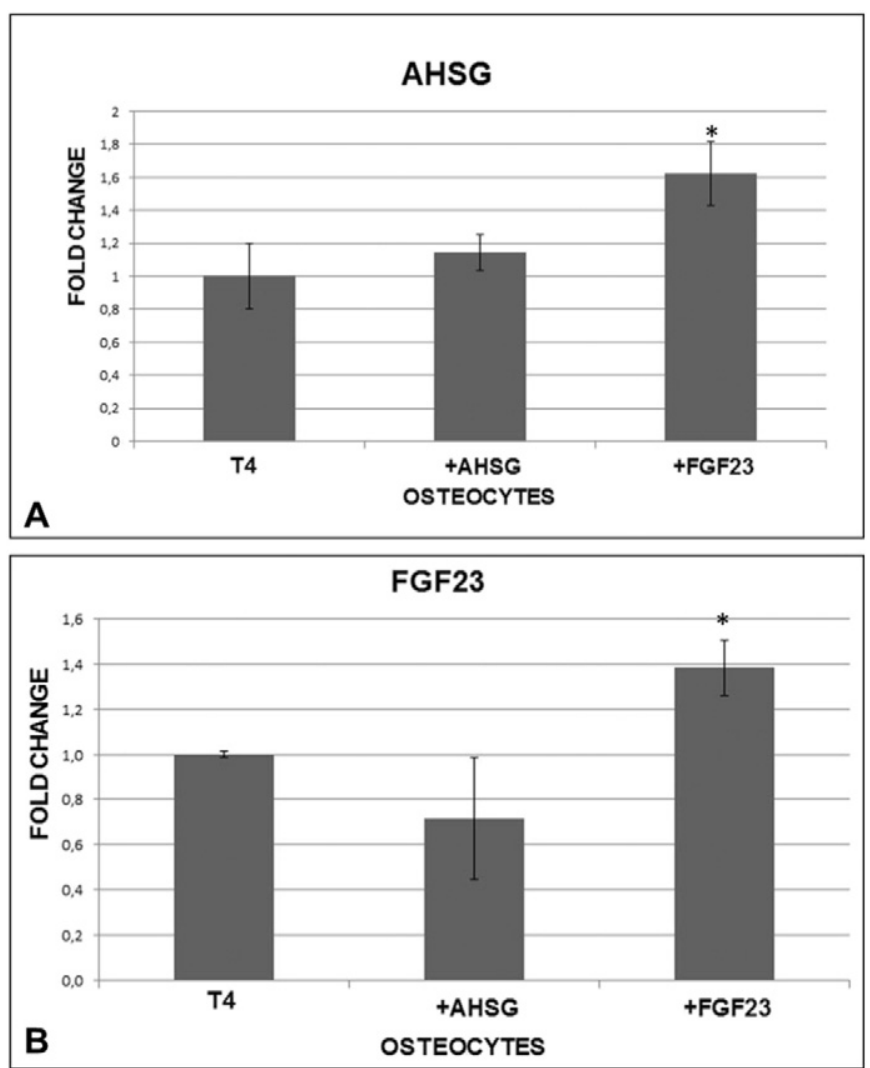

Fig. 4. A. Changes of AHSG mRNA expression $24 \mathrm{~h}$ after the addition of either FGF23 or AHSG to T4 Os. Bars are means \pm SD; $n=3-4$ /group. B. Changes of FGF23 mRNA expression $24 \mathrm{~h}$ after the addition of either FGF23 or AHSG to T4 Os. Bars are means \pm SD; $n=3$ / group. Asterisks indicate significant differences versus Os T4 (ctrl): ${ }^{*}=\mathrm{p}<0.05$ by ANOVA test.

on both AHSG mRNA and protein production (Fig. 10A, B). After FGFRs blockade alone, a striking increase of AHSG mRNA and protein was observed. In contrast, FGF23 overexpression in the presence of FGFRs block was not additive to the AHSG stimulatory effects (Fig. 10A, B).

\subsection{AHSG promoter gene activation during FGF23 overexpression}

To test if the effects of FGF23 overexpression are dependent on genomic effects, we assessed the changes of AHSG promoter gene luciferase activity. FGF23 overexpression translated into a stimulatory effect on AHSG promoter gene, which was consistently unchanged over the 48 hour observation time. At variance, the FGFRs block resulted in an even more marked, though less persistent, stimulatory effect on AHSG promoter gene. FGF23 overexpression in the presence of FGFRs blockade activated AHSG promoter, an effect which persisted over $48 \mathrm{~h}$ (data not shown) but that was not additive to the AHSG stimulatory effects (Fig. 11A, B).

We also explored whether FGF23 was present either in the cytoplasm and/or the nucleus. As shown by qualitative WB analysis, FGF23 protein was present in the cytoplasmic and, to a lesser extent, in the nuclear compartments (Fig. 11C).

\subsection{Cell colocalization and protein interaction of AHSG and FGF23}

We first detected the colocalization of AHSG and FGF23 by double immunofluorescence staining, which was clearly evident in our cells (Fig. 12A, B, C). In order to verify the proximity of these two proteins, we performed Duolink assay which provides signal interaction of proteins which must be within 0 to $40 \mathrm{~nm}$ distance. Results obtained 

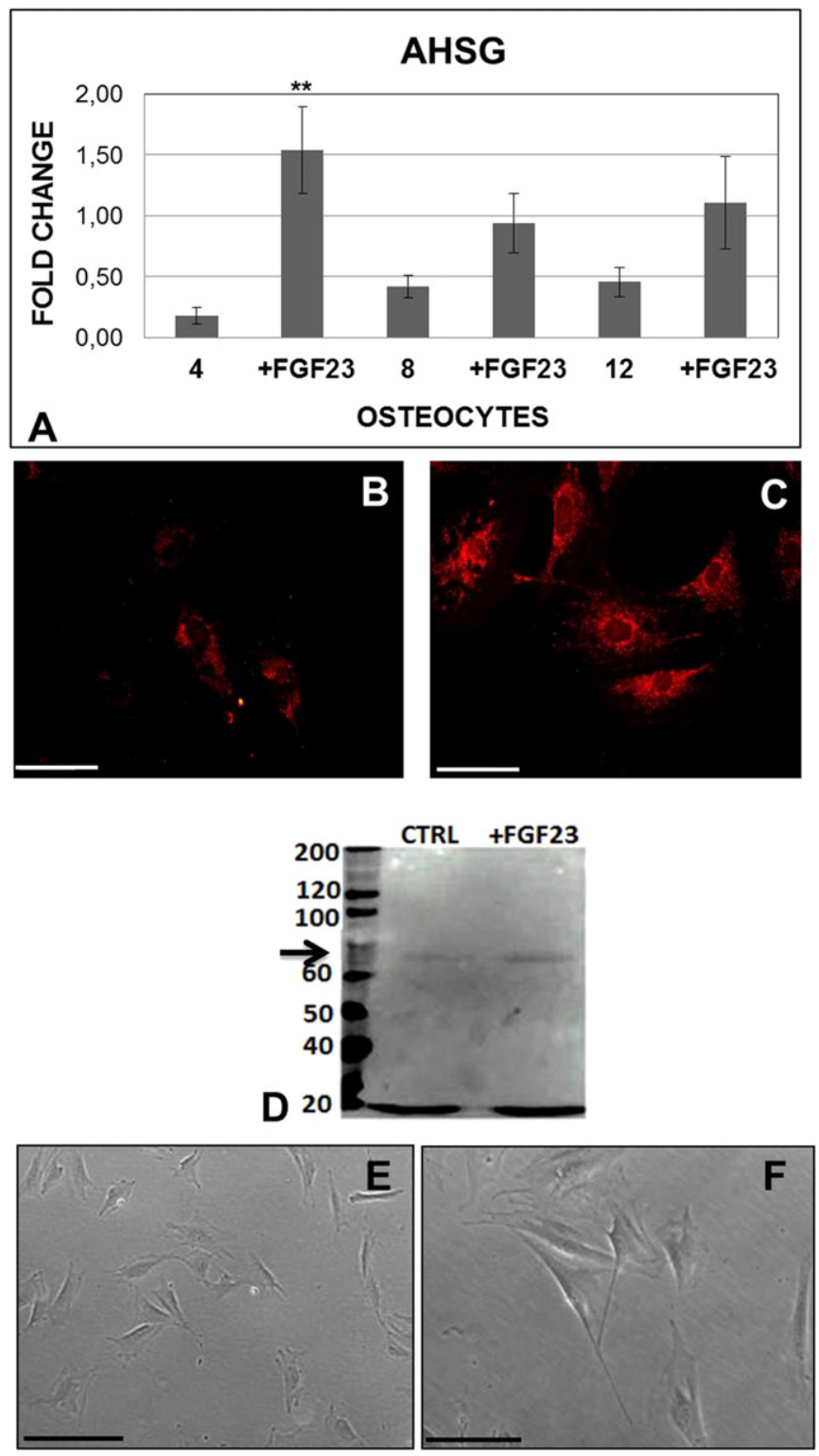

Fig. 5. A. AHSG mRNA expression in Os cultured for 4, 8, 12 days in either control conditions or following FGF23 treatment. Bars are means \pm SD; $n=3-4$ /group. B, C. Immunofluorescence of AHSG at T4 in control (B) and in FGF23-treated (C) Os. Scale bars: $100 \mu \mathrm{m}$. D. WB analysis of AHSG at T4 in control and in FGF23-treated Os; $n=3$ /group. Cofilin (bottom of the gel) acted as a loading control. E, F. Light microscopy in control (E) and FGF23-treated (F) T4 Os. Scale bars: $100 \mu \mathrm{m}$. Asterisks indicate significant differences versus Os $\mathrm{T} 4$ (ctrl): ${ }^{* *}=\mathrm{p}<0.01$ by ANOVA.

using this approach show that AHSG and FGF23 appeared very close to each other. AHSG and FGF23 interaction was also determined and quantified in both nucleus and in cytoplasm of Os T4 cells. AHSG/FGF23 interaction was evident both in the cytoplasm and, to a lesser extent, in the nuclear space (Fig. 13A-D).

\section{Discussion}

Among its multifold roles, the bone is the subject and the object of an intense hormonal and metabolic control, being involved not only in its long time recognized canonical functions (mechanical, protective, hematopoietic, MM) but also in the more recently described interactions with the CV system $[22,27,28]$.

All these functions rely on a complicated system which recognizes the Os as the central key player, since this cell, through its multi-branched
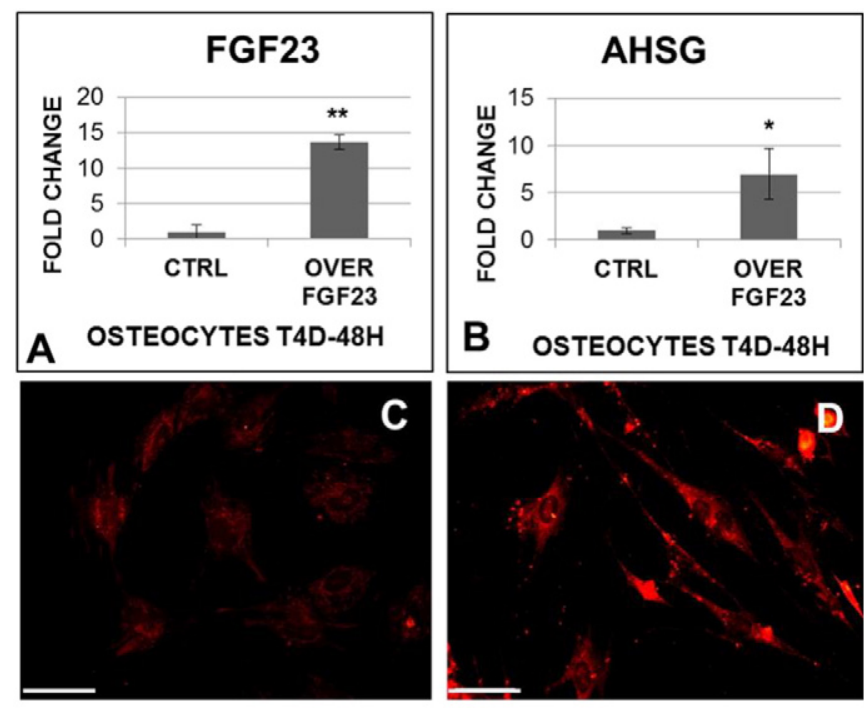

CTRL OVER EXP.

FGF23
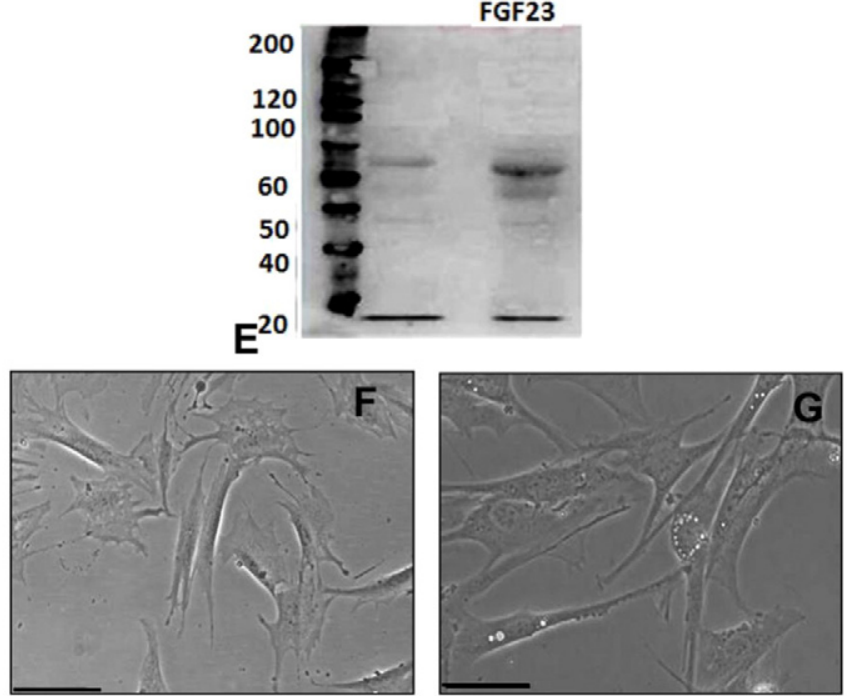

Fig. 6. A. qRT PCR of FGF23 mRNA in control and FGF23 overexpressing Os at $48 \mathrm{~h}$. Bars are means $\pm S D ; n=4$ /group. B. qRT PCR of AHSG mRNA in control and FGF23 overexpressing Os at 48 h. Bars are means $\pm S D ; n=4 /$ group. C, D. Immunofluorescent detection of AHSG protein at $48 \mathrm{~h}$ in control (C) and FGF23 overexpressing (D) Os. Scale bars: $100 \mu \mathrm{m}$. E. AHSG WB analysis in control and FGF23 overexpressing Os at $48 \mathrm{~h} . \mathrm{n}=3$ /group. Cofilin (bottom of the gel) acted as a loading control. F, G. Light microscopy of control (F) and FGF23 overexpressing Os at $48 \mathrm{~h}(\mathrm{G})$. Scale bars: $100 \mu \mathrm{m}$. Asterisks indicate significant differences versus Os T4 (ctrl): ${ }^{*}=\mathrm{p}<0.05 ;^{* *}=\mathrm{p}<0.01$ Student's t-test.

canaliculi web, controls the $\mathrm{Ob}$ and osteoclast (Oc) functions by juxta and paracrine mechanisms, via the production of a great number of hormonal factors, namely FGF23, osteocalcin, osteoprotegerin, sclerostin, Dickkopf-related protein 1(DKK1), and bone morphogenic proteins 2-7 (BMP2-7), leading to metabolic effects on many different organs (kidney, brain, liver, pancreas, heart, vascular system, etc.) [29,30].

In these dynamic systems we focused our attention on two proteins which have been believed to play roles in both the bone and vascular systems, namely FGF23 and AHSG, the first being a well-recognized bone-derived protein and the second being produced mainly by the liver, though it has been suggested that it is produced by bone cells too. We explored this issue using our recently described method for in vitro culturing of stable Os cells [24]. We utilized the MC3T3-E1 cell line to obtain Os in an easy and highly reproducible way, to have the opportunity of culturing cells at different stages of maturation (from preosteoblast to mature $\mathrm{Ob}$ and then to Os) from the same cell lineage, 

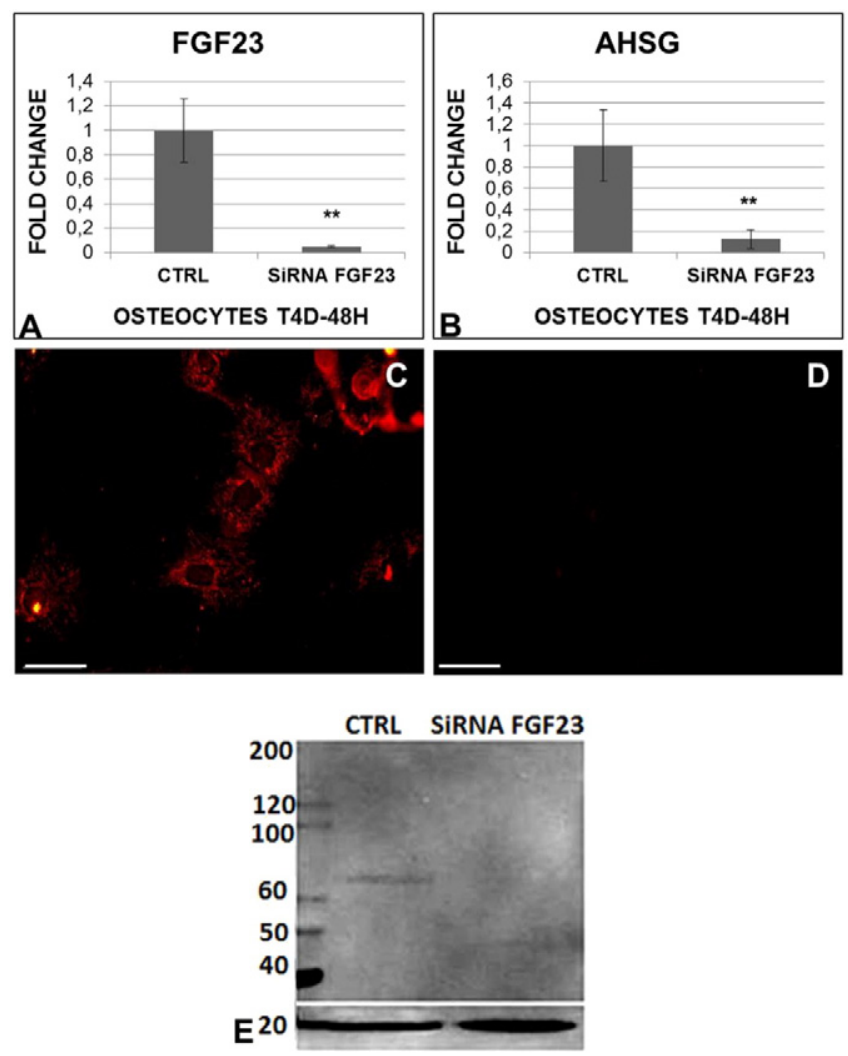

Fig. 7. A. qRT PCR of FGF23 mRNA in control and silenced Os at $48 \mathrm{~h}$ after FGF23 gene silencing. Bars are means $\pm \mathrm{SD} ; \mathrm{n}=3$ /group. B. qRT PCR of FGF23 mRNA in control and silenced Os at $48 \mathrm{~h}$ after FGF23 gene silencing. Bars are means $\pm \mathrm{SD} ; \mathrm{n}=3$ /group. $\mathrm{C}, \mathrm{D}$. Immunofluorescent detection of AHSG protein in control (C) and in FGF23 mRNA silenced Os (D) $48 \mathrm{~h}$ after gene silencing. Scale bars C, D: $100 \mu \mathrm{m}$. E. AHSG WB in T4 control and FGF23 mRNA silenced Os; $n=3$ /group. Cofilin (bottom of the gel) acted as a loading control. Asterisks indicate significant differences versus Os T4 (ctrl): ${ }^{* *}=\mathrm{p}<0.01$ by Student's t-test. and to generate mature Os in the complete absence of extracellular matrix allowing these cells for unlimited biological applications (e.g. electrophysiology studies).

First, we reconfirmed our previous results demonstrating that the cultured cells represent well differentiated Os owing to the high levels of sclerostin. These cells also produce FGF23 at a consistently higher degree when compared to other Ob cells. The maturation status of these cells was also confirmed by the specific IS for FGF23 which was confined to Os cells in mouse tibial bone tissue.

It is still a matter of discussion whether AHSG is really produced in bone cells or is just captured by the bone microenvironment from its circulating pool [8-13]. More recently, Coen and co-workers [21] confirmed that AHSG mRNA and protein were expressed in some Os, in the $\mathrm{Ob}$, though at a variable degree, and in the mineralized matrix in bone biopsies from hemodialysis patients.

In our study, IHC in mouse tibial bone clearly demonstrated that AHSG was highly expressed in Os, while it was not evident in the majority of Ob. Furthermore, IHC performed on our cell cultures, confirmed the expression of AHSG in the Os which clearly increased over time, while $\mathrm{Ob}$ seem to produce a limited amount of this protein and its levels vanishing over time. These results are consistent with an in situ production of AHSG by bone cells, mainly by Os. This concept is reinforced by the demonstration that both AHSG gene (mRNA) and protein (WB) expression progressively and markedly increased in Os. These findings were paralleled by the demonstration of a consistent release of AHSG (ELISA) in the supernatant of both Os and Ob cultures, though again this was, by far, more evident in the former. So, our study confirms that AHSG is indeed produced by bone cells themselves, in particular by Os. Furthermore, our findings also demonstrate that AHSG production is variable both in Ob and in Os depending on the stage of cell maturation, with a progressive decrease and increase in the former and in the latter respectively. This finding might in part explain the discrepancies of the reported AHSG expression in bone cells between different studies.

On the basis of these initial observations, we went on to investigate if there was a direct interaction between FGF23 and AHSG in our bone cell cultures.
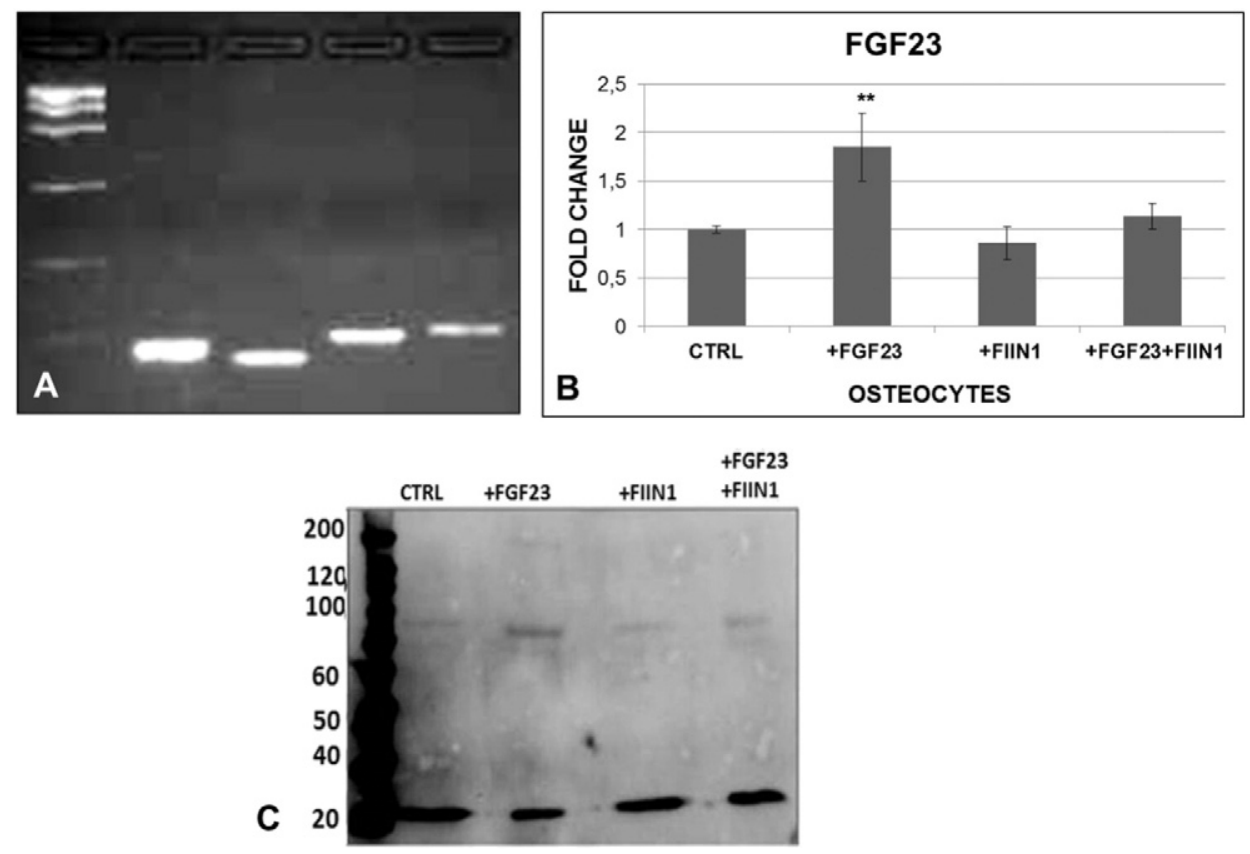

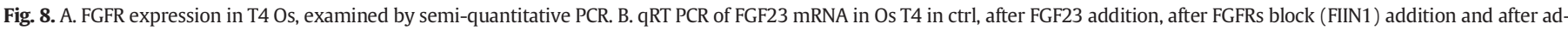

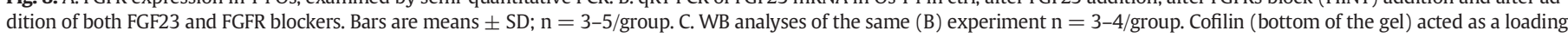
control. Asterisks indicate significant differences versus Os T4 (ctrl): ${ }^{* *}=\mathrm{p}<0.01$ by ANOVA. 

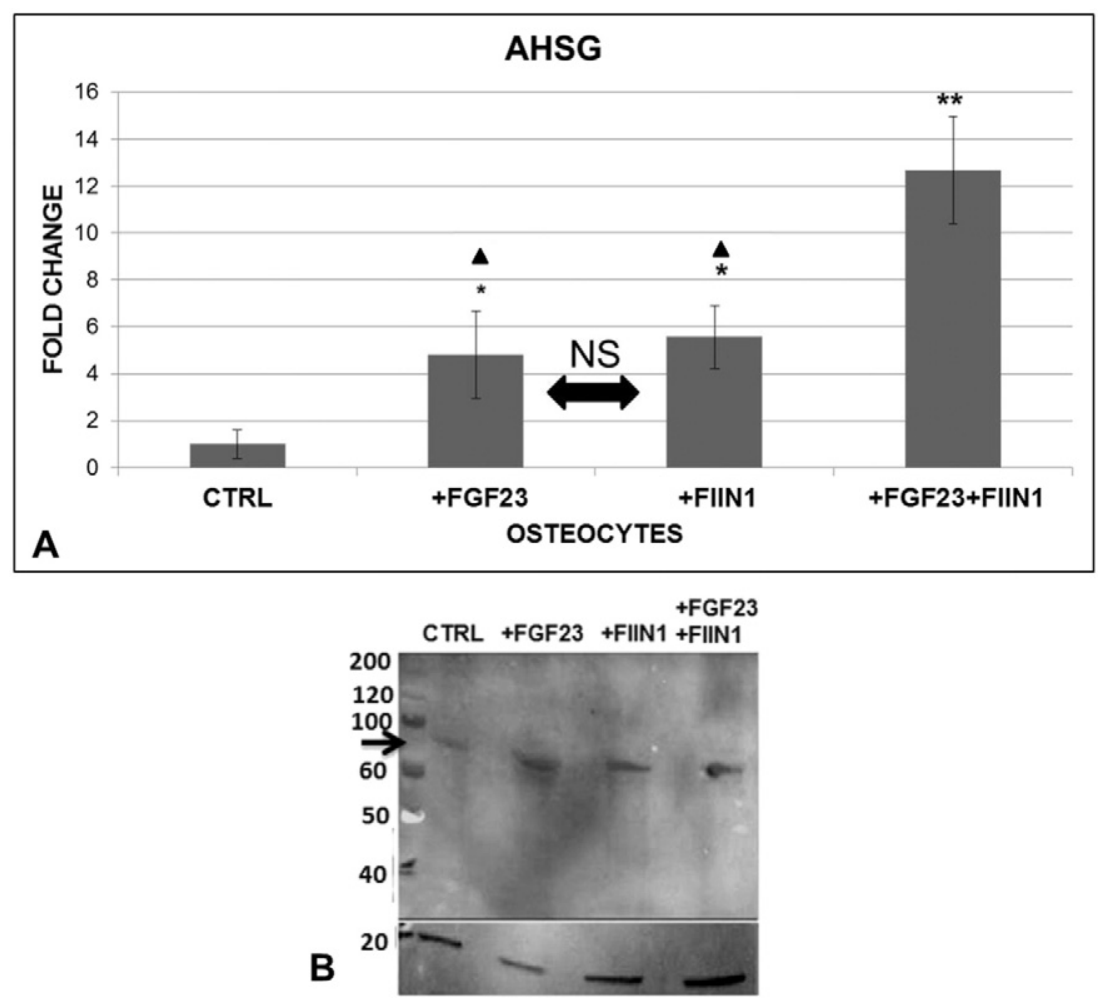

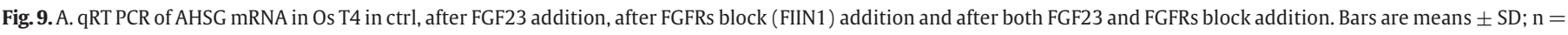

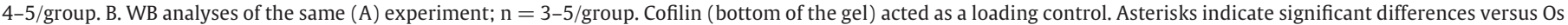
T4 (ctrl): ${ }^{*}=\mathrm{p}<0.05,{ }^{* *}=\mathrm{p}<0.01$. Triangles significant differences versus FGFRs blocked plus FGF23 treated Os: $\boldsymbol{\Delta}=\mathrm{p}<0.05$ by ANOVA.

Preliminarily to any experimental study, we verified by Gene Cards® data base whether FGF23 and AHSG recognize the same or different transcription factors (TFs) in the same animal species as the one used in our experiments (mice). We found no evidence of common TF was albeit some direct and indirect TF interactions are reported [Gene Cards ${ }^{\circledR}$ ].
Subsequently, we wondered if the addition of recombinant FGF23 or AHSG to cell supernatant could impact on FGF23 and/or AHSG production. For this purpose, we chose to investigate this issue in cultured Os at T4, which is the time when these cells initiate their production of both proteins. The addition of FGF23 induced a consistent increase in
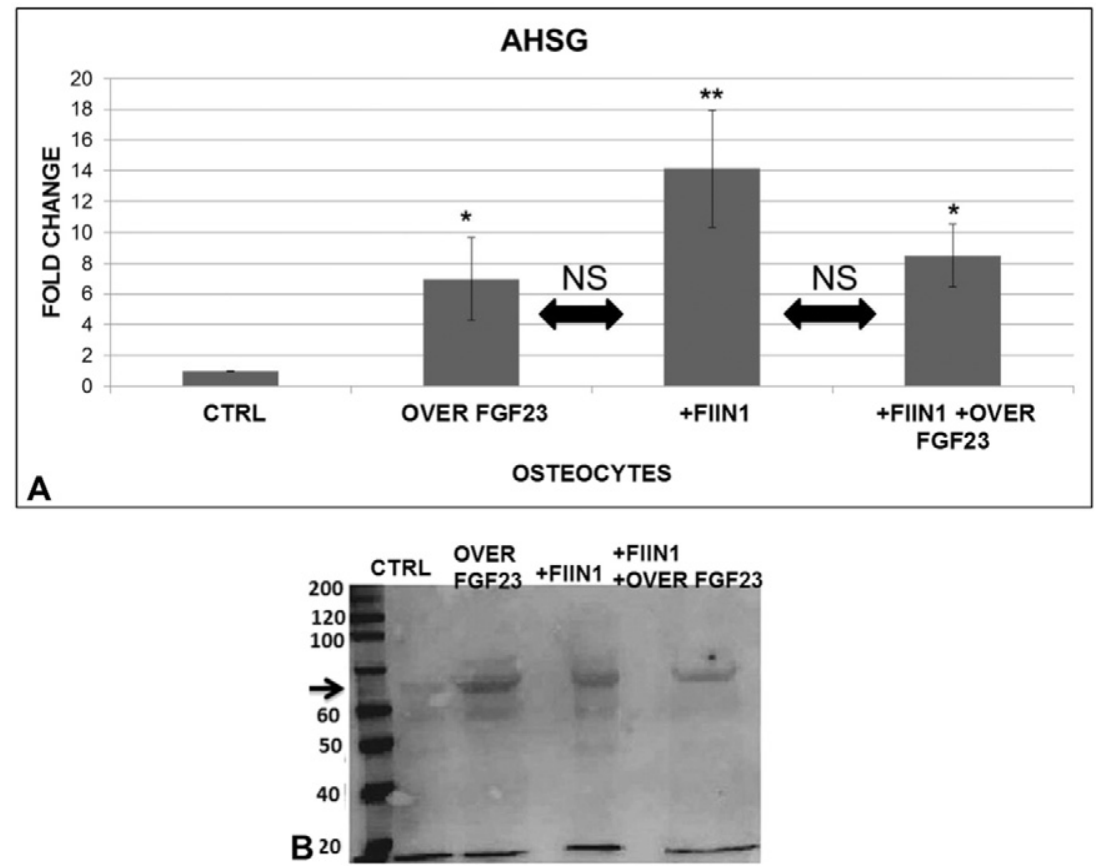

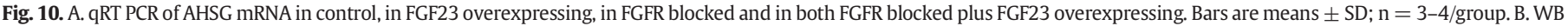

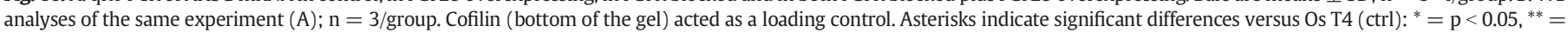
$\mathrm{p}<0.01$ by ANOVA. 

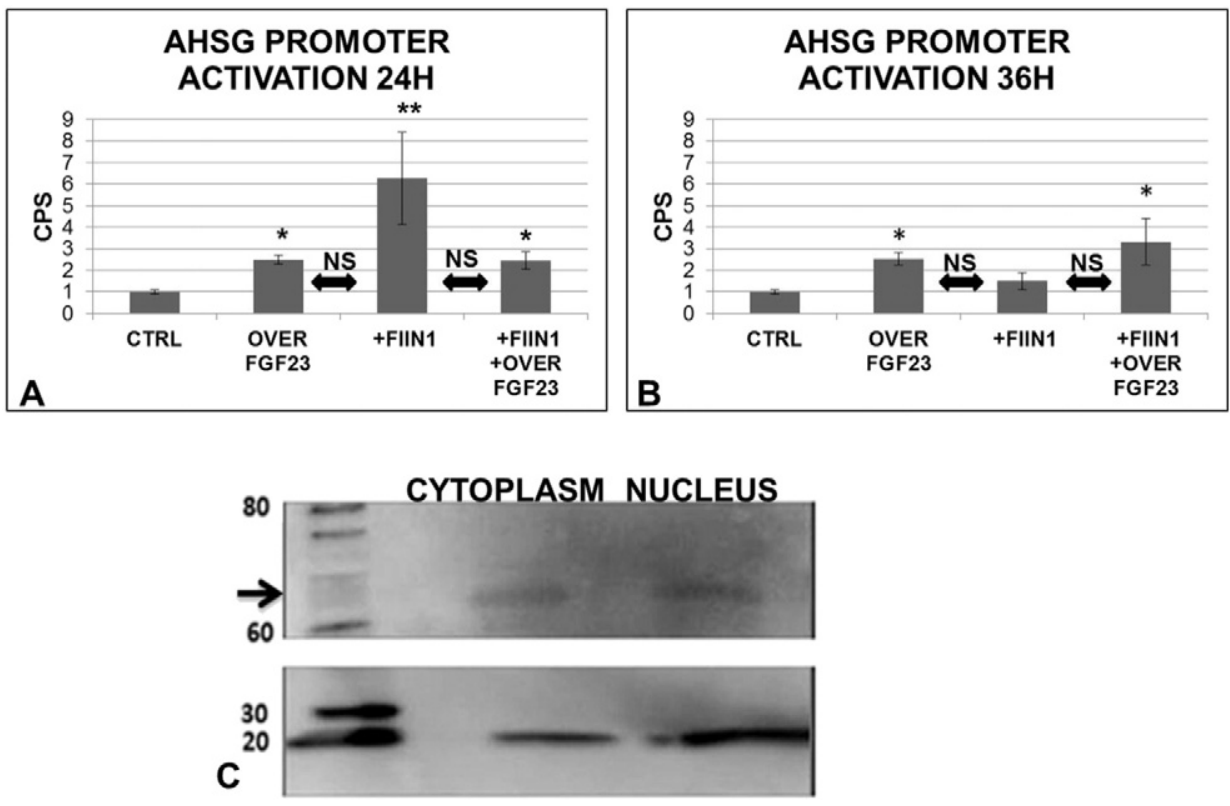

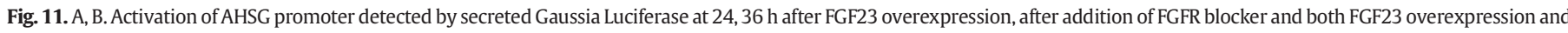

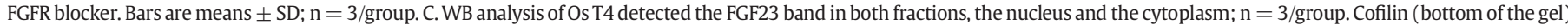
acted as a loading control. Asterisks indicate significant differences versus Os T4 (ctrl): ${ }^{*}=\mathrm{p}<0.05,{ }^{* *}=\mathrm{p}<0.01$ by ANOVA.

both FGF23 itself and AHSG production, while AHSG addition was not followed by any variation of either protein expression.

Though it is known that in bone cells [27,31] FGF23 production is stimulated by other FGFs, to the best of our knowledge this is the first report of an auto-stimulatory effect of FGF23.

The meaning of this observation is currently unclear and deserves further specific studies. One striking finding is the unexpected stimulatory effect of FGF23 on AHSG. Thus, we studied the FGF23 effects on AHSG production challenging Os with recombinant FGF23 at several time points (days 4, 8, 12 in culture). The largest and most significant increase in AHSG production was confirmed only in Os cultured for 4 days, while this enhanced AHSG expression did not reach significance in cells cultured for 8 or 12 days. Interestingly, our study shows that at the same time of maximal AHSG production, Os start to change their shape, producing their dendritic processes.

From these data we can argue that FGF23 could play a role in controlling AHSG production by Os, but this effect is probably active only in the first stages of Os maturation.
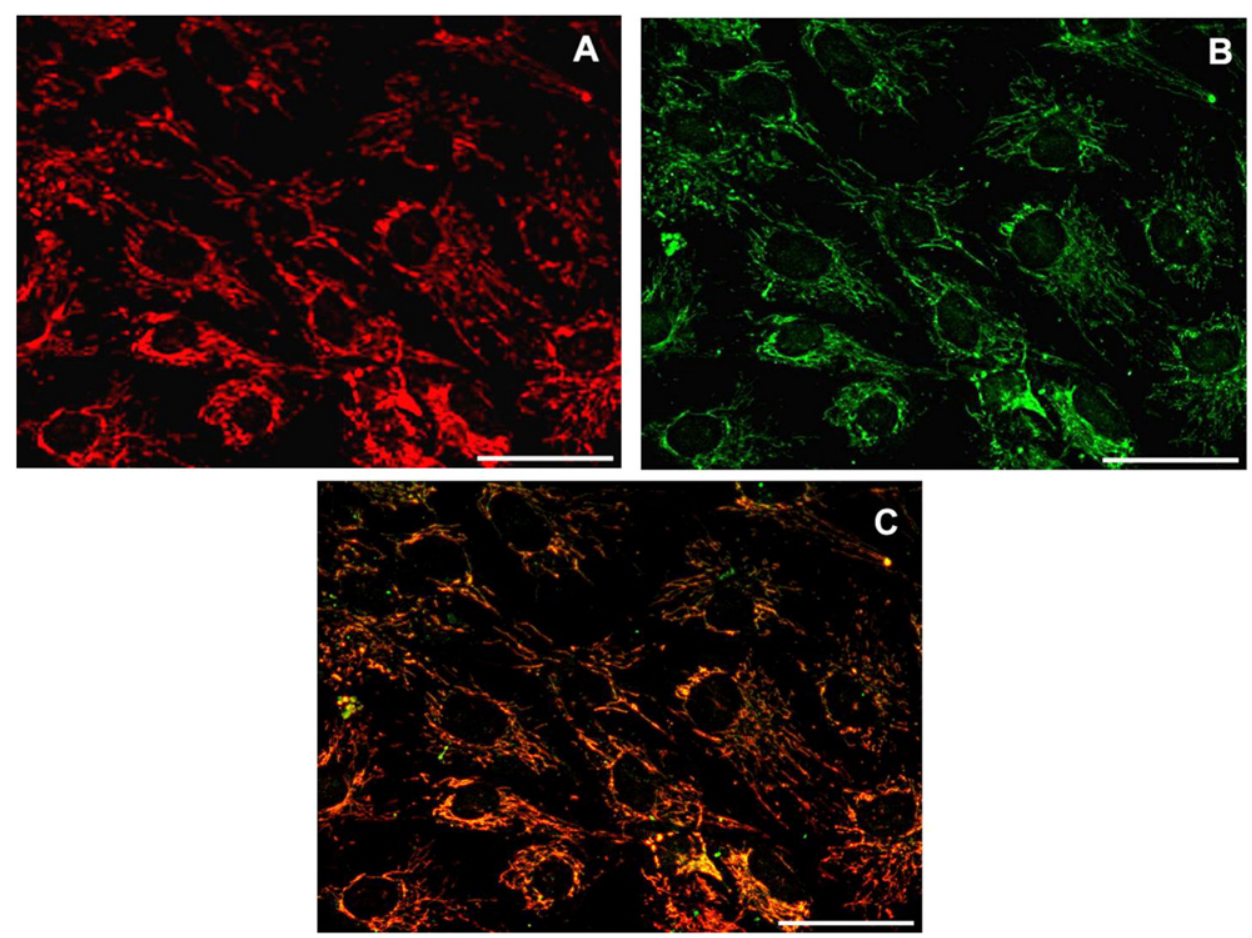

Fig. 12. Immunofluorescence of AHSG (A), FGF23 (B) and merge (C) shows their colocalization. Scale bars: $50 \mu \mathrm{m}$. 

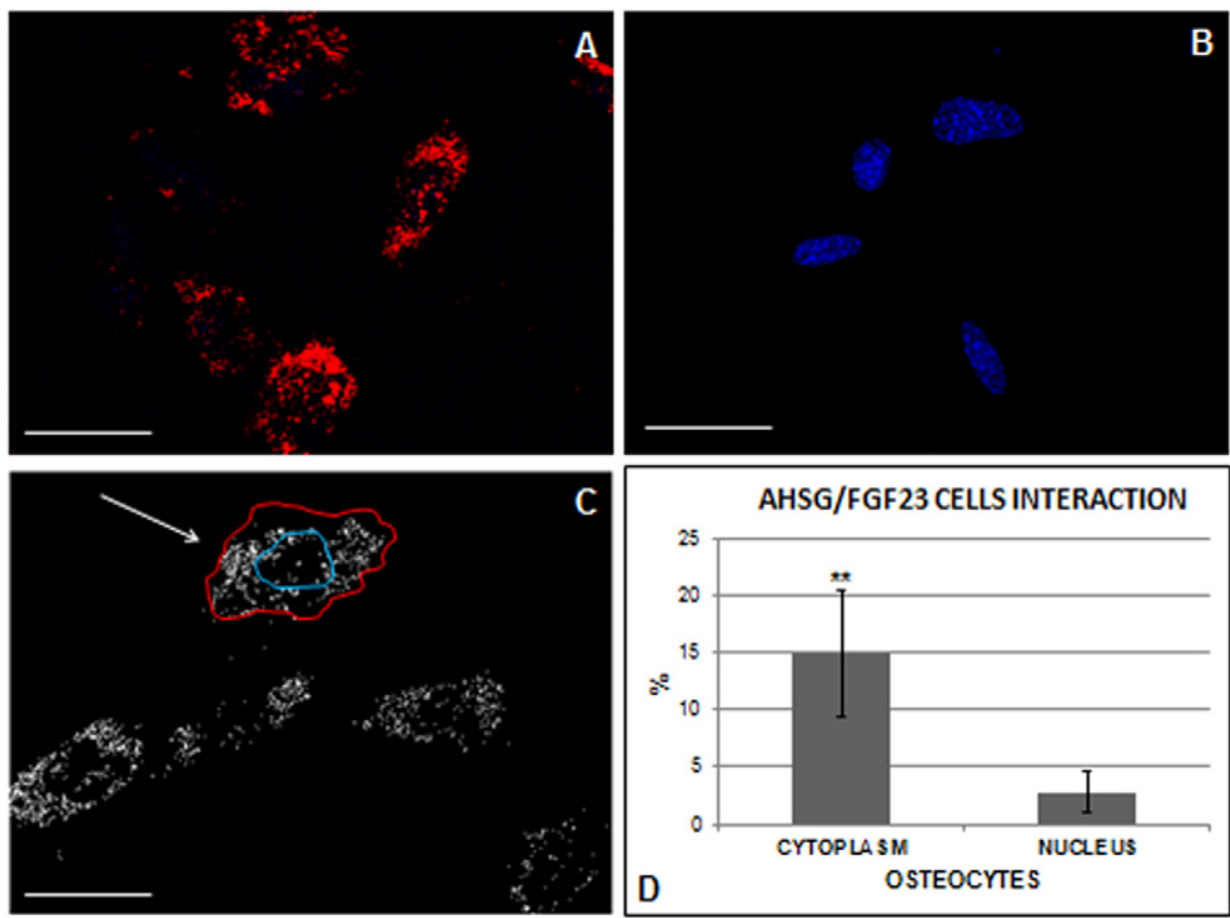

AHSG/FGF23 CELLS INTERACTION

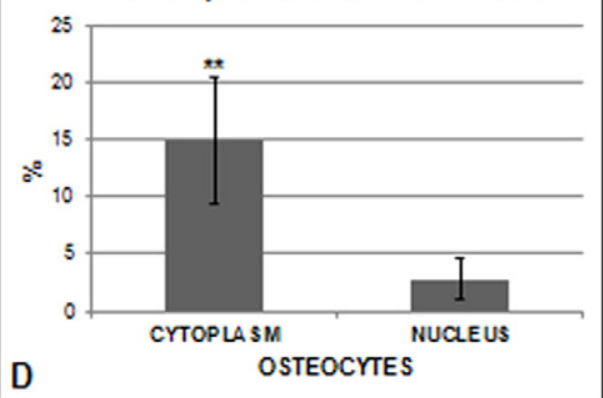

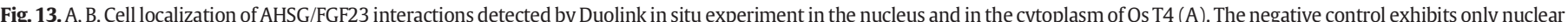

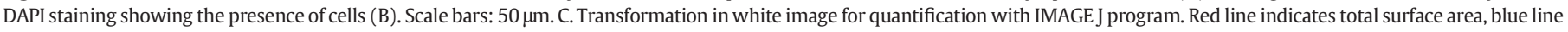

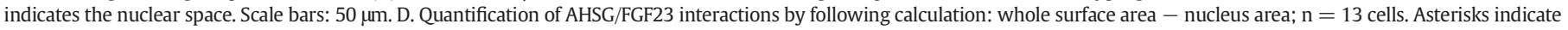
significant differences ${ }^{* *}=\mathrm{p}<0.01$ by Student's t-test.

There is no clear explanation for the meaning of such a stimulatory effect of FGF23 on AHSG production in bone cells. It could be just hypothesized that an autocrine and paracrine production of AHSG in the stage of Os maturation could facilitate the elongation of dendritic processes of these cells into the mineralizing matrix.

Since the stimulus driven by high extracellular FGF23 concentration could be considered non-physiological or, at best, a rare condition, we moved on to verify if FGF23 overexpression in Os, which mimics the overproduction of this protein independently of the nature of the stimulating factor, could yield the same effects. Again, FGF23 overexpression in Os resulted in a significant increase of AHSG mRNA and protein expression in line to what previously observed after exogenous FGF23 addition to the cells. In parallel, an even more apparent elongation of Os overexpressing FGF23 was observed as compared to that seen in cells stimulated with exogenous FGF23. This finding is reminiscent of that already reported in Os stimulated by other FGFs [32,33]. Since it is known that FGFs can also stimulate FGF23 production [34], it would be interesting to know if the shape modification of Os after FGFs stimulation is a direct or indirect, FGF23-mediated, effect.

We then went on to explore if FGF23 silencing in Os, which mimics the reduced production from any stimuli, is also associated with changes in AHSG production. Our results show that in the Os downregulation of FGF23 mRNA was mirrored by a concomitant reduction in both AHSG mRNA and protein. Interestingly, FGF23 mRNA silencing was accompanied by a recovery in AHSG protein the rate of which was faster than that of FGF23 protein. These results strengthen the importance of the controlling effect of FGF23 on AHSG production in Os, which can be rapidly reverted with even minor changes in FGF23 levels.

In a next series of experiments, we wondered if the observed effects of FGF23 were mediated by its recognized 4 receptors (FGFRs). First, we confirmed that our cultured cells express all the 4 classical FGFRs (FGFR1, FGFR2, FGFR3 and FGFR4), with FGFR1 appearing as the most expressed receptor, as already described in Os [35,36].
Then we tested the effect of blocking FGFRs in all the above described experimental conditions. We chose FIIN1 as a FGFRs blocker, since its irreversible effects guarantee the stability of the block throughout the duration of the experimental conditions [35]. However, it is also worth underlining that the FIIN1 does not equally block all the 4 FGFRs, with its maximal effect having been reported on FGFR1 and the least on FGFR4 [35,36].

The addition of FIIN1 in control conditions did not produce any detectable effect on both FGF23 mRNA and protein, excluding any independent effect of the FGFRs block on the basal production of this hormone. Of relevance, the auto-stimulatory effect of FGF23 addition to the culture medium was completely abolished by FGFRs inhibition, strongly suggesting that this effect is completely mediated by FGFRs activity.

It is acknowledged that FGF23 requires Klotho as an essential coreceptor, in order to for exert its effects $[37,38]$. Since Klotho is not expressed in bone tissue, the exact pathway by which FGF23 can produce the observed effects in our bone cells remains to be clarified. We could hypothesize that, given the high concentrations of FGF23 achieved in the peri-cellular space of secreting cells, a nonspecific binding of FGFRs might occur, as has been suggested for the effects of this hormone on non-classical target organs in CKD patients, where very high peripheral concentrations of FGF23 are often observed. Alternatively, the existence of other as yet undefined FGF23 receptors could be hypothesized.

In contrast with the results on FGF23 production, FGFRs inhibition by itself translated into an unexpected upregulation of both AHSG mRNA and protein expression levels. Furthermore, when FGF23 stimulus was superimposed to the FGFRs inhibition an additional and consistent increase of AHSG mRNA and, to a lesser extent, of protein were observed. We have no clear explanation for these findings, though one can hypothesize that FIN1 might have a direct effect on intracellular pathways controlling mRNA translational and/or post-translational 
mechanisms. Alternatively, we cannot exclude that FIIN1 might inhibit some other receptor(s), with a basic inhibitory effect on the AHSG production, which, when blocked, produces a stimulatory effect. However, neither of the two above hypotheses can explain the additional stimulatory effect of FGF23, in the presence of FGFRs blockage. We can only speculate that this additional stimulatory effect of FGF23 might be dependent on the activation of other yet unrecognized receptors. Alternatively it could also be hypothesized that, given that FIN1 is not equally potent on all the 4 FGFRs, the stimulatory effect of FGF23 on AHSG production might be driven mainly by the FGFRs less inhibited by FIIN1 blockade. Further investigations are necessary to explore this issue further.

The results of FGFRs inhibition on FGF23 overexpression effects, besides confirming the stimulatory effect of FGFRs block per se on AHSG production, demonstrate that the increase of FGF23 stimulates AHSG production in an FGFRs independent way, though this effect was not additive with FGFRs blockade per se as observed in the preceding experimental set.

These results were paralleled by concomitant changes of AHSG promoter luciferase activity in these experimental conditions. Furthermore, WB analysis confirmed that FGF23 was present also at nuclear level, though to a lesser extent than in the cytoplasm. Even more relevant, we found that FGF23 and AHSG colocalized both at cytoplasm and nuclear level. Since these results were obtained with the duo-link techniques, which is positive for an intermolecular distance lower than $40 \mathrm{~nm}$, they indirectly suggest that these two proteins might be closely linked to each other. Overall, these results suggest that the described effects might be mediated by common genomic pathways. However, as previously mentioned, though no common TF at FGF23 and AHSG gene level have been reported, some indirect and direct interactions have been described. Therefore we cannot completely exclude that the described genomic effect of FGF23 on AHSG promoter could be dependent on some direct interaction(s) utilizing shared pathways (e.g. PPAR-y, CEBP- $\alpha$ ) [GeneCards $®$ data base]. Clearly, this point deserves further investigation.

\section{Conclusions}

Our results strongly support the hypothesis that bone cells are a specific site for production of AHSG. Furthermore, our data also show that FGF23 is consistently involved in the control of AHSG production in the Os. These effects are mainly evident in the earlier stages of Os maturation, at least in our experimental settings, suggesting that bone AHSG production could play a role in counteracting and/or slowing the calcifying process within the bone mineral matrix, possibly facilitating the development of Os dendritic processes. Our study also suggests that the pathways for FGF23-dependent AHSG stimulation could be different when FGF23 acts as an autocrine factor (as it is mimicked by over-expression) or as a paracrine or hormonal stimulus (as shown by FGF23 addition into the medium). Though our results provide clear evidence for an FGFR dependence of the auto stimulatory effect of FGF23 on its own production, they do not completely clarify the role of FGFRs in the FGF23-dependent AHSG production, an issue which mandates further investigations. Importantly, we also demonstrated a colocalization of FGF23 and AHSG at both cytoplasmic and nuclear level, suggesting also a close functional co-operation between these two molecules. A number of open issues ensue from these initial results. First, which are the specific roles, if any, of Os AHSG production in the bone; second, if these effects are limited to the bone tissue; third, if the FGF23 control of AHSG secretion, this could also affect circulating levels of this protein. Finally, due to the well-known involvement of both FGF23 and AHSG on CV and kidney functions, it would be worth knowing if and how much this newly described pathway might affect these organs and systems. Future studies are expected to give more insights into these issues.

\section{Methods}

\subsection{Cell culture conditions}

The MC3T3-E1 cell line, subclone 4 [39,40] established from normal newborn mouse calvaria, was obtained from ATCC (LGC Standards S.r.l., Sesto San Giovanni, Milan, Italy). Cells were cultured in alpha-MEM medium (Invitrogen, Milan, Italy), supplemented with 10\% FBS (SigmaAldrich, Milan, Italy) and 1\% streptomycin/penicillin (Sigma-Aldrich) in $75-\mathrm{cm}^{2}$ flasks at a density of 400000 cells $/ \mathrm{cm} 2$. When cells reached $80 \%$ confluence, $50 \mathrm{mg} / \mathrm{ml}$ ascorbic acid and $3 \mathrm{mM}$ glycerol 2-phosphate disodium salt hydrate (Sigma-Aldrich) were added to the medium. Medium was changed every other day. After 5 days of incubation with ascorbic acid-glycerol phosphate (AA/GP), the cells were trypsinized and replated at a density of 7000 cells $/ \mathrm{cm}^{2}$ and cultured with basal medium plus AA/GP and $10 \mu \mathrm{M}$ retinoic acid (All Trans Retinoic Acid, ATRA, from Sigma-Aldrich) to obtain Os [24,25,41]. Alternatively cells were cultured with basal medium plus AA/GP to obtain Ob. The cell populations were then studied at several time points, namely after 4, 8, 12 days of treatment.

\subsection{Immunohistochemistry and immunocytochemistry}

In this study ten male 45 day Balb/c mice (Charles River Italy, Inc. Lecco) were used. They were acclimatized for one week and water and basal diet were given ad libitum. All experiments were conducted in accordance with the EU Directive 2010/63/EU for animal experiments.

Calvaria tibial tissues were Isolated from 45 day old male mice by treatment with decalcifying solution ( $\mathrm{HCl} 37 \% /$ Formic acid 85\%/distilled water) for $6 \mathrm{~h}$ after which they were fixed in PFA 4\% and embedded in paraffin. Dewaxed tissue sections were incubated with an rabbit anti-fibroblast growth factor 23 [Swiss Prot: Q9GZV9] (Santa Cruz Biotechnology, Heidelberg, Germany) and mouse anti-Alpha-2-HSglycoprotein [Swiss Prot: P02765] (Abcam). Then IHC was performed on tissue sections using broad spectrum horse-radish peroxidase (HRP) polymer conjugated (Invitrogen). Four IHC cells were plated on Petri dishes or coverslips, where the cells were cultured in the same conditions as described above. For indirect immunofluorescence, cells were fixed in either $4 \%$ paraformaldehyde or cold acetone, depending on the primary antibody to be applied. After fixation, cells were incubated with the primary antibodies rabbit anti-FGF23 (Santa Cruz Biotechnology), and/or goat anti-Alpha-2-HS-glycoprotein [Swiss Prot: P02765] (Santa Cruz Biotechnology). As secondary, fluorescentlylabeled antibodies, the following were used: Alexa Fluor 546 goat antirabbit IgG, Alexa Fluor 546 rabbit anti-goat IgG highly cross adsorbed (Invitrogen).

Specificity of Ab labeling was demonstrated by the lack of staining after substituting the primary antibody with control immunoglobulins (rabbit primary, mouse primary or goat $\mathrm{Ab}$ isotype controls from Invitrogen). Slides were mounted with Fluorsave aqueous mounting medium (Calbiochem, Merck Chemicals Ltd., Nottingham, UK). Images were acquired by a Zeiss Axioscope 40FL microscope, equipped with AxioCam MRc5 digital videocamera and immunofluorescence apparatus (Carl Zeiss SpA, Arese, Mi, Italy), and recorded by AxioVision software 4.3 (Carl Zeiss SpA) or by Zeiss AxioObserver microscope equipped with high resolution digital videocamera (AxioCam, Zeiss) and Apotome system for structured illumination, and recorded by AxioVision software 4.8 .

\subsection{ELISA tests}

AHSG levels in the supernatants were evaluated using a mouse ELISA AHSG kit (Uscnk, Houston, USA), according to the manufacturer's instructions. Protein expression was normalized using Janus Green Whole-Cell Stain for $5 \mathrm{~min}$. Careful washing was followed by addition of Elution Buffer and absorbance was read at $615 \mathrm{~nm}$. Replicate 
background measurements were subtracted to all $450 \mathrm{~nm}$ measures. The resulting $450 \mathrm{~nm}$ values were then normalized to the $615 \mathrm{~nm}$ values to account for differences in numbers of cells.

The minimum detectable dose of mouse AHSG is typically less than $3.64 \mathrm{ng} / \mathrm{ml}$

FGF23 levels in the supernatants were evaluated by mouse FGF23 C-terminal ELISA kit (Immutopics International, Pantec, Torino, Italy), according to the manufacturer's instructions. Mouse FGF23 has a sensitivity of $4 \mathrm{pg} / \mathrm{ml}$, intra-assay variation of $6.2 \%$ and inter assay variation of $5.9 \%$.

\subsection{FGF23 and AHSG addition}

For studies of reciprocal effects of the addition of FGF23 and AHSG, Os 4 days were stimulated with $400 \mathrm{PG} / \mathrm{ml}$ of human recombinant FGF23 (Immunological Sciences, Roma, Italy) or with $500 \mu \mathrm{G} / \mathrm{ml}$ of bovine serum AHSG (Sigma-Aldrich) for $24 \mathrm{~h}$. To determine the effects of recombinant FGF23 addition on AHSG expression, Os were stimulated with $400 \mathrm{PG} / \mathrm{ml}$ of FGF23 for $24 \mathrm{~h}$ at 4, 8, 12 days in culture, as specified.

\subsection{FGF23 overexpression}

4 day Os were transfected with 10 nM FGF23 mouse cDNA clone (ORF with GFP tagged $C$ terminal) (OriGene Technologies Inc.MD, USA) using Lipofectamine2000 (Invitrogen) as transfecting agent. As control, non-targeting cDNA clone was employed at the same concentration. Mouse FGF23 ORF cDNA clone with GFP tagged $C$ terminal [Vector: pCMV6_AC-GFP, Tag: C-terminal TurboGFP, Sequence Data: [GenBank: NM_022657.3]. ORF Size: 756 bp, restriction sites: Sgfl-Mlul.

\subsection{FGF23 silencing}

4 day Os were transfected with $10 \mathrm{nM}$ siRNA duplexes using Lipofectamine2000 (Invitrogen) as the transfecting agent. Three commercially available siRNAs complementary to FGF23 mRNA were used (SigmaAldrich). First sequence forward 5' GCU AUC ACC UAC AGA UCC A 3' reverse primer 5' UGG AUC UGU AGG UGA UAG C 3', second sequence forward primer 5' CCA UAG GGA UGG UCA UGU A 3' reverse primer 5' UAC AUG ACC AUC CCU AUG G 3', third sequence forward primer $5^{\prime}$ CUC GAA GGU UCC UUU GUA U 3', reverse primer 5' AUA CAA AGG AAC CUU CGA G $3^{\prime}$. As a control, non-targeting siRNAs were transfected at the same concentration.

Transfection efficiency was determined by a fluorescently-tagged siRNA (Alexa-Fluor488; Amersham, Perkin Elmer, Waltham, MA).

\subsection{FGFRs inhibition}

The FGFRs inhibitor FIIN1 hydrochloride (1 uM, Tocris Biosciences, Bristol UK) was administered to Os 4 days for $24 \mathrm{~h}$. When used in the experiments with FGF23 stimulation, this inhibitor was added $6 \mathrm{~h}$ before the addition of FGF23 and then together with FGF23.

\section{8. mRNA extraction, RT-PCR, and $q R T-P C R$}

Total RNA of Ob was extracted by TRIzol (Invitrogen) and precipitated by chloroform-isopropyl alcohol, washed in ethanol 75\%, resuspended in nuclease free-water, then treated with DNase, resuspended, and quantified by spectrophotometry cDNA was prepared from $1 \mu \mathrm{g}$ RNA using the iScript Select cDNA Synthesis Kit and oligo(dt)20 primers (Bio-Rad, Segrate, Milan, Italy). While total RNA of Os was extracted by Aurum total RNA mini kit (Biorad).

Half of the mRNA extracted was used to evaluate AHSG expression, with the following primers: AHSG [GenBank: NM_001276450]: forward 5' CAC CGA ACT TAC CAC GAC CT 3'; reverse 5' ATG TCC TGT CTG CCA AAA CC 3'; and the other half was used to evaluate FGF23 expression, with the following primers: FGF23 [GenBank: AF263536]: forward 5'
GAT CCC CAC CTC AGT TCT CA 3'; reverse 5' CCG GAT AGG CTC TAG CAG TG 3'; data were normalized against the expression of GAPDH [GenBank: AY618199]: forward primer 5' GGT ATC GTG GAA GGA CTC ATG A 3'; reverse primer 5' GGC CAT CCA CAG TCT TCT G 3'. After assessment of primer specificity by RT-PCR, Real Time RT-PCR was run with iQ Sybr Green Supermix (Bio-Rad) on a MylQ instrument (Bio-Rad), and data were analyzed by the IQ5 Bio-Rad Software. For RT-PCR of FGFrs Os 4 days cDNA was amplified by PCR using primers FGFr1 [GenBank: NM_001079909]: forward primer 5' AGA CGG ACA ACA CCA AAC CA 3'; reverse primer 5' CCG CAT GCA GTT TCT TCT CC 3'; FGFr2 [GenBank: EF143340]: forward primer 5' CCG GGG AAT CGC TAG AGT TG 3'; reverse primer 5' GTG CAC CCC ATC CTT AGT CC 3'; FGFr3 [GenBank: NM_001163216]: forward primer 5' GTG GTG GCA GCT GTG ATA CT 3'; reverse primer 3' TTA AGC GGG AAG CGA GAG AC 5'; FGFr4 [GenBank: NM_008011]: forward primer 5' CTT GGG ACT TCA CAT CCC C 3'; reverse primer 5' AGA GCT GAT GCC CCT TTC AC 3'. A single amplicon of the correct size was generated with each primer set. Amplification conditions were PCR buffer with $2.5 \mathrm{mM} \mathrm{MgCl}_{2}, 200 \mathrm{nM}$ of each primer and fast Start Taq in the presence of $200 \mathrm{nM}$ dNTPs (Roche). Samples were amplified for 40 cycles at $95{ }^{\circ} \mathrm{C}$ for $30 \mathrm{~s}$, an annealing step at $60{ }^{\circ} \mathrm{C}$ for $30 \mathrm{~s}$ and an extension step at $72{ }^{\circ} \mathrm{C}$ for $30 \mathrm{~s}$ with a final extension at $72{ }^{\circ} \mathrm{C}$ for $10 \mathrm{~min}$. PCR products were resolved with loading dye on $2 \%$ agarose gels containing $5 \mu$ ethidium bromide in $0.5 \times$ Tris Borate EDTA (TBE) buffer alongside a low molecular weight DNA ladder and photographed under UV light using a GelDoc system (BioRad).

\subsection{Protein isolation and WB}

Cells were lysed by complete Lysis-M kit (Roche Monza, Italy). For the separation of cellular cytoplasm and nuclear fractions, a second lysis was performed using a solution containing $60 \mu \mathrm{l}$ of Lysis-M and $10 \mu \mathrm{l}$ of $\mathrm{NaCl}$ to obtain nuclear proteins.

Protein lysates of cytoplasm or nucleus were then separated on a SDS-PAGE and transferred by electroblotting on a PVDF membrane (ImmunBlot PVDF membrane, Bio-Rad). After blocking, each membrane was incubated with the primary antibodies, rabbit anti-FGF23 (Santa Cruz Biotechnology) and mouse anti-Fetuin-A (Abcam), followed by the HRP-conjugated secondary antibodies, and positive reaction products were identified by chemiluminescence (BM Chemiluminescence WBting Kit, Roche). Loading controls were conducted with antibodies directed against rabbit anti-cofilin [Swiss Prot: P18630] (Abcam).

Images were digitally acquired by Chemidoc XRS instrument (BioRad) and analyzed by Quantity One software (Bio-Rad).

\subsection{Gaussia Luciferase activity}

Control Os T4 were first transfected with Gluc-SEAP dual reporter clone pre-designed for AHSG promoter (Secrete-Pair Dual Luminescence and Gaussia Luciferase Reporter Assay kit, Genecopeia, Catalogue No.: MPRM17140-PG04) to study the basic AHSG promoter activities. Subsequently Os T4 were transfected with Gluc-SEAP dual reporter in combination with $10 \mathrm{nM}$ FGF23 mouse cDNA clone (ORF with C terminal GFP tagged, OriGene Technologies Inc.MD, described in Section 5.5) to analyze the effect of FGF23 overexpression on AHSG promoter activity. To determine the role of endogenous FGF23 we conducted the same two described above experiments in the presence of the FGFRs inhibitor FIIN1 $(1 \mu \mathrm{M})$. Every transfection was performed using Lipofectamine2000 (Invitrogen) as transfecting agent.

Culture medium from transfected Os was collected to study the promoter activities at 24, 36, $48 \mathrm{~h}$ after transfection.

For the detection of the promoter activation, secreted luciferase was evaluated by bioluminescence analyzed with spectrofluorometers FLX-Xenius (SAFAS Monaco). Seap (secreted alkaline phosphatase) was used as the internal control for signal normalization. 


\subsubsection{Clone information}

GenBank: NM_013465, Description: Promoter clone for gene AHSG, Promoter length: $1241 \mathrm{bp}$, Sequences length upstream of TSS: $1190 \mathrm{bp}$, Sequence length downstream of TSS: 50 bp, Vector: pEZX-PG04, Antibiotic: Kanamycin, Reporter Gene:Gluc, Cloning Site at 5': BgIII, EcoRI, Whole Plasmid size: 7952 bp, Stable selection Marker: Puromycin, Tracking Gene: secreted alkaline phosphatase (SEAP), Cloning Site at $3^{\prime}$ :HindIII. Suggested Sequencing Primers suggested: forward primer 5' AGTTACTTAAGCTCGGGCCC 3'; reverse primer 5' TTGTTCTCGGTGGG CTTGGC $3{ }^{\prime}$.

\subsection{Protein interaction}

To detect AHSG-FGF23 interactions, Duolink (Sigma-Aldrich) in situ experiment was performed. Cells were fixed in cold acetone and incubated with rabbit anti-FGF23 primary antibody (Santa Cruz Biotechnology) and mouse anti-Fetuin-A (Abcam). Subsequently a second incubation with relative PLUS/MINUS PLA probe was performed and after amplification by ligation to visualize the interaction by immunofluorescence. Quantification of AHSG/FGF23 interactions was evaluated using IMAGE J program by following calculation: whole surface cells area - nucleus area.

\subsection{Statistical analyses}

Experiments were conducted on at least 3 replicates per each condition and time point. Data were expressed as mean \pm standard deviation (SD), Student's t-test and ANOVA were applied to determine significance $(\mathrm{p}<0.05)$. For $\mathrm{qRT}-\mathrm{PCR}$, relative RNA abundance was determined using the comparative Ct method [42]. The fold-change (FC) calculated by the software, was evaluated according to the following formula: $\sigma \mathrm{FC}=\mathrm{FC} * \ln 2 * \operatorname{sqrt}(\sigma \mathrm{x} 2 / \mathrm{nx}+\sigma \mathrm{y} 2 / \mathrm{ny})$. Fold change error bars, represent the standard deviation $(\sigma)$ of the fold change (FC).

$\mathrm{p}$ values were calculated based on Student's t-test of the replicate $2^{\wedge}$ ( - Delta $\mathrm{Ct}$ ) values for each gene in the control group and treatment groups, and $\mathrm{p}$ values $<0.05$ were considered significant.

\section{Competing interests}

The authors have declared that no competing interests exist.

\section{Authors' contributions}

MD: carried out the experiments, participated in the study design, interpreted the results and participated in the manuscript writing.

RMP: helped in the study design, experiment coordination, and cooperated in the interpretation of results.

IM: carried out light microscopy.

AS: participated in bioluminescence study.

PC: assisted in conceiving images.

GL: helped in culture cell experiments.

LM: helped in culture cell experiments.

ACM: participated in the statistical analyses and result interpretation. RA: participated in the statistical analyses and result interpretation.

RD: participated in the result discussion.

MP: designed the study, supervised the experiments, interpreted the results and wrote the manuscript.

All authors read and approved the final manuscript.

\section{Acknowledgments}

Funding was provided by IRCCS Ospedale Maggiore Policlinico.

\section{References}

[1] K.O. Pedersen, Fetuin, a new globulin isolated from serum, Nature 154 (1944) 575.
[2] I.R. Dickson, A.R. Poole, A. Veis, Localisation of plasma alpha2HS glycoprotein in mineralising human bone, Nature 256 (5516) (1975) 430-432.

[3] B.A. Ashton, J. Höling, J.T. Triffitt, Plasma proteins present in human cortical bone: enrichment of the $\alpha_{2}$ HS-glycoprotein, Calcif. Tissue Res. 22 (1976) 27-33.

[4] A. Heiss, A. DuChesne, B. Denecke, J. Grötzinger, K. Yamamoto, Structural basis of calcification inhibition by alpha 2-HS glycoprotein/Fetuin-A. Formation of colloidal calciprotein particles, J. Biol. Chem. 278 (2003) 13333-13341.

[5] C. Schafer, A. Heiss, A. Schwarz, R. Westenfeld, M. Ketteler, J. Floege, W. MullerEsterl, T. Schinke, W. Jahnen-Dechent, The serum protein alpha 2-HeremansSchmid glycoprotein/fetuin-A is a systemically acting inhibitor of ectopic calcification, J. Clin. Invest. 112 (2003) 357-366.

[6] G. Schlieper, R. Westenfeld, V. Brandenburg, M. Ketteler, Inhibitors of calcification in blood and urine, Semin. Dial. 20 (2007) 113-121.

[7] C. Binkert, M. Demetriu, S. Baltra, M. Szweras, H.C. Tenenbaum, W. Dennis, Regulation of osteogenesis by fetuin-a, The journal of biochemical chemistry. 274 (1999) 28514-28520.

[8] G.C. Colclasure, W.S. Lloyd, M. Lamkin, W. Gonnerman, R.F. Troxler, G.D. Offner, W Bürgi, K. Schmid, R.B. Nimberg, Human serum alpha 2HS-glycoprotein modulates in vitro bone resorption, J. Clin. Endocrinol. Metab. 66 (1) (1988) 187-192.

[9] T. Schinke, C. Amendt, A. Trindl, O. Pöschke, W. Müller-Esterl, W. Jahnen-Dechent, The serum protein alpha2-HS glycoprotein/fetuin-a inhibits apatite formation in vitro and in mineralizing calvaria cells. A possible role in mineralization and calcium homeostasis, J. Biol. Chem. 271 (34) (1996) 20789-20796.

[10] O. Nakamura, J.A. Kazi, T. Ohnishi, N. Arakaki, Q. Shao, T. Kajihara, Y. Daikuhara, Effects of rat fetuin-a on stimulation of bone resorption in the presence of parathyroid hormone, Biosci. Biotechnol. Biochem. 63 (8) (1999) 1383-1391.

[11] J.T. Triffitt, U. Gebauer, B.A. Ashton, M.E. Owen, J.J. Reynolds, Origin of plasma alpha 2HS-glycoprotein and its accumulation in bone, Nature 262 (5565) (1976) 226-227.

[12] T. Ohnishi, N. Arakaki, O. Nakamura, S. Hirono, Y. Daikuhara, Purification, characterization, and studies on biosynthesis of a 59-kDa bone sialic acid-containing protein (BSP) from rat mandible using a monoclonal antibody. Evidence that 59-kDa BSP may be the rat counterpart of human alpha 2-HS glycoprotein and is synthesized by both hepatocytes and osteoblasts, J. Biol. Chem. 266 (22) (1991) 14636-14645.

[13] G. Coen, P. Ballanti, G. Silvestrini, et al., Immunohistochemical localization and mRNA expression of matrix Gla protein and fetuin-A in bone biopsies of hemodialysis patients, Virchows Arch. 454 (2009) 263-271.

[14] J. Xie, M.J. Baumann, L.R. McCabe, Adsorption of serum fetuin-a to hydroxylapatite does not contribute to osteoblast phenotype modifications, J. Biomed. Mater. Res. A 73 (1) (2005) 39-47.

[15] J.Q. Feng, et al., Loss of DMP1 causes rickets and osteomalacia and identifies a role for osteocytes in mineral metabolism, Nat. Genet. 38 (11) (2006) 1310-1315.

[16] H. Wang, et al., Overexpression of fibroblast growth factor 23 suppresses osteoblast differentiation and matrix mineralization in vitro, J. Bone Miner. Res. 23 (6) (2008) 939-948.

[17] Sitara, D., et al., Genetic evidence of serum phosphate-independent functions of FGF-23 on bone. PLoS Genet.20084(8):p. e1000154.

[18] O. Andrukhova, U. Zeitz, R. Goetz, M. Mohammadi, B. Lanske, R.G. Erben, FGF23 acts directly on renal proximal tubules to induce phosphaturia through activation of the ERK1/2-SGK1 signaling pathway, Bone 5 (3) (2012) 621-628.

[19] B.L. Clarke, FGF23 regulation of phosphorus homeostasis is dependent on PTH, Endocrinology 152 (11) (2011) 4016-4018.

[20] P. Messa, FGF23 and vascular calcifications: another piece of the puzzle? Nephrol. Dial. Transplant. 29 (8) (2014) 1447-1449.

[21] G. Coen, P. DE Paolis, P. Ballanti, et al., Peripheral artery calcifications evaluated by histology correlate to those detected by CT: relationships with fetuin-A and FGF-23, J. Nephrol. 24 (3) (2011) 313-321.

[22] S.L. Dallas, M. Prideaux, L.F. Bonewald, The osteocyte: an endocrine cell and more, Endocr. Rev. 34 (5) (2013) 658-690.

[23] L.F. Bonewald, M.J. Wacker, FGF23 production by OS, Pediatr. Nephrol. 28 (4) (2013) 563-568.

[24] D. Mattinzoli, P. Messa, A. Corbelli, M. Ikehata, C. Zennaro, S. Armelloni, M. Li, L. Giardino, M.P. Rastaldi, A novel model of in vitro osteogenesis induced by retinoic acid treatment, Eur. Cell. Mater. 24 (2012) 403-425.

[25] D. Mattinzoli, P. Messa, A. Corbelli, M. Ikehata, A. Mondini, C. Zennaro, S. Armelloni, M. Li, L. Giardino, M.P. Rastaldi, Application of retinoic acid to obtain osteocytes cultures from primary mouse osteoblasts, J. Vis. Exp. 87 (2014).

[26] L.F. Bonewald, M.J. Wacker, FGF23 production by osteocytes, Pediatr. Nephrol. 28 (4) (2013) 563-568

[27] M.G. Vervloet, Z.A. Massy, V.M. Brandenburg, S. Mazzaferro, M. Cozzolino, P. UreñaTorres, J. Bover, D. Goldsmith, Bone: a new endocrine organ at the heart of chronic kidney disease and mineral and bone disorders, Lancet Diabetes Endocrinol 2 (5) (2014) 427-436.

[28] K. Sharan, V.K. Yadav, Hypothalamic control of bone metabolism, Endocrinol. Metab. 28 (5) (2014) 713-723.

[29] M. Capulli, R. Paone, N. Rucci, Osteoblast and osteocyte: games without frontiers, Arch. Biochem. Biophys. 561 (2014) 3-12.

[30] J.J. Kazama, M. Fukagawa, In and out of the bone: can the osteocyte escape skeletal jail and yet regulate mineralization? Kidney Int. 85 (1) (2014) 11-12.

[31] C.M. Teven, E.M. Farina, J. Rivas, R.R. Reid, Fibroblast growth factor (FGF) signaling in development and skeletal diseases, Genes Dis 1 (2) (2014) 199-213.

[32] I. Allodi, L. Casals-Díaz, E. Santos-Nogueira, F. Gonzalez-Perez, X. Navarro, E. Udina FGF-2 low molecular weight selectively promotes neuritogenesis of motor neurons in vitro, Mol. Neurobiol. 47 (2) (2013) 770-781.

[33] R.R. Gupta, D.J. Yoo, C. Hebert, C. Niger, J.P. Stains, Induction of an osteocyte-like phenotype by fibroblast growth factor-2, Biochem. Biophys. Res. Commun. 402 (2) (2010) 258-264. 
[34] H. Terai, et al., Activation of the FGF2-FGFR1 autocrine pathway: a novel mechanism of acquired resistance to gefitinib in NSCLC, Mol. Cancer Res. 11 (7) (2013) 759-767.

[35] Y. Takei, T. Minamizaki, Y. Yoshiko, Functional diversity of fibroblast growth factors in bone formation, Int. J. Endocrinol. 2015 (2015) 729352

[36] W. Zhou, W. Hur, U. Mc Dermott, A. Dutt, W. Xian, S.B. Ficarro, J. Zhang, S.V. Sharma, J. Brugge, M. Meyerson, J. Settleman, N.S. Gray, A structure-guided approach to creating covalent FGFR inhibitors, Chem. Biol. 17 (3) (2010) 285-295.

[37] I. Urakawa, Y. Yamazaki, T. Shimada, K. Iijima, H. Hasegawa, K. Okawa, T. Fujita, S. Fukumoto, T. Yamashita, Klotho converts canonical FGF receptor into a specific receptor for FGF23, Nature 444 (2006) 770-774.

[38] H. Kurosu, Y. Ogawa, M. Miyoshi, M. Yamamoto, A. Nandi, K.P. Rosenblatt, M.G. Baum, S. Schiavi, M.C. Hu, O.W. Moe, M. Kuro-o, Regulation of fibroblast growth factor-23 signaling by klotho, J. Biol. Chem. 281 (10) (2006) 6120-6123.
[39] D. Wang, K. Christensen, K. Chawla, G. Xiao, P.H. Krebsbach, R.T. Franceschi, Isolation and characterization of MC3T3-E1 preosteoblast subclones with distinct in vitro and in vivo differentiation/mineralization potential, J. Bone Miner. Res. 14 (1999) 893-903.

[40] L.D. Quarles, D.A. Yohay, L.W. Lever, R. Caton, Wenstrup RJ distinct proliferative and differentiated stages of murine MC3T3-E1 cells in culture: an in vitro model of osteoblast development, J. Bone Miner. Res. J7 (1992) 683-692.

[41] H. Nagasawa, S. Takahashi, A. Kobayashi, H. Tazawa, Y Tashima, Sato K effect of retinoic acid on murine preosteoblastic MC3T3-E1 cells, J. Nutr. Sci. Vitaminol. 51 (2005) 311-318.

[42] K.J. Livak, T.D. Schmittgen, Analysis of relative gene expression data using real-time quantitative PCR and the 2(-delta delta C(T)) method, Methods 25 (2001) 402-408. 